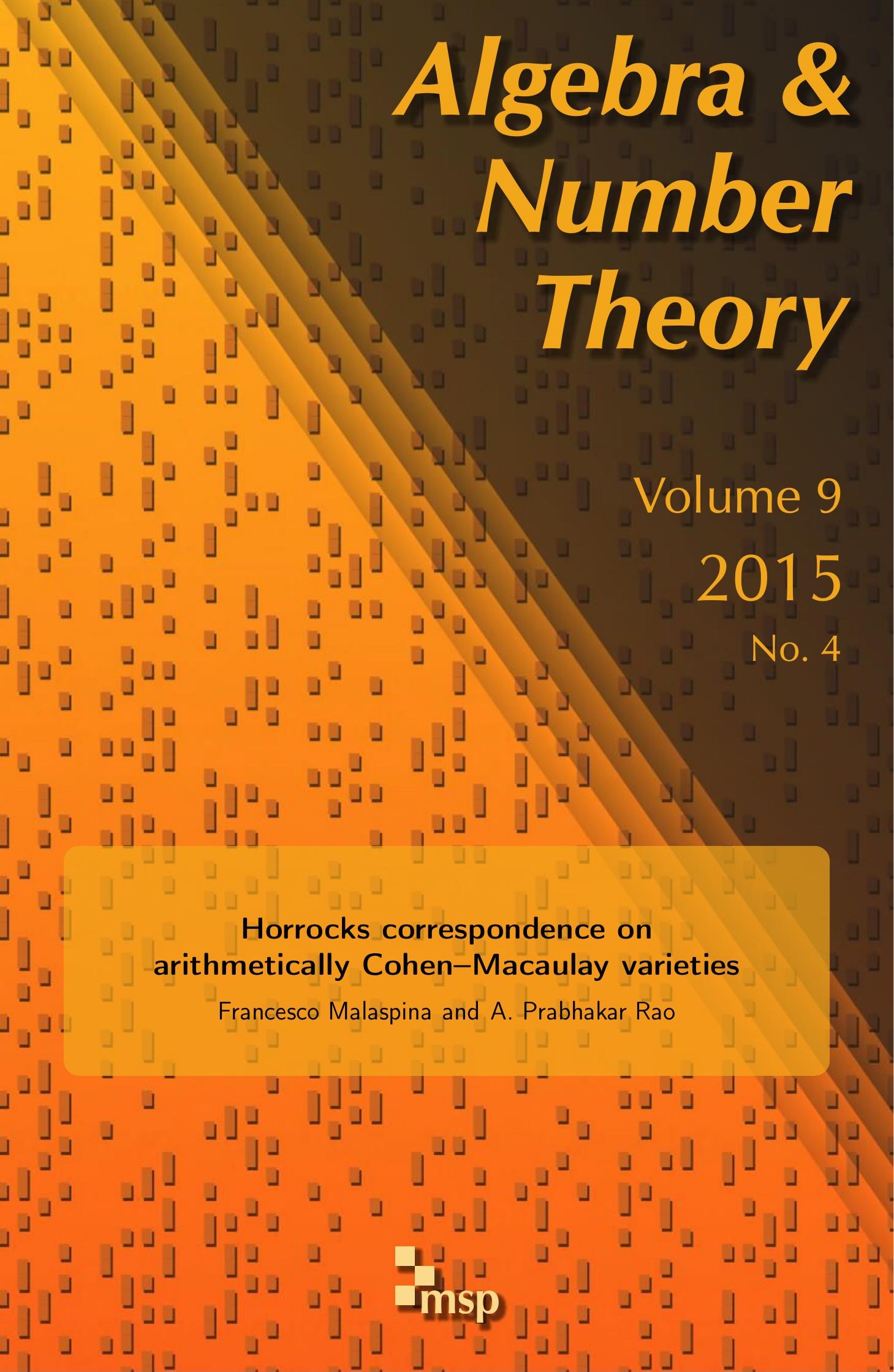




\title{
Horrocks correspondence on arithmetically Cohen-Macaulay varieties
}

\author{
Francesco Malaspina and A. Prabhakar Rao
}

\begin{abstract}
We describe a vector bundle $\mathscr{E}$ on a smooth $n$-dimensional arithmetically CohenMacaulay variety in terms of its cohomological invariants $H_{*}^{i}(\mathscr{E}), 1 \leq i \leq n-1$, and certain graded modules of "socle elements" built from $\mathscr{E}$. In this way we give a generalization of the Horrocks correspondence. We prove existence theorems, where we construct vector bundles from these invariants, and uniqueness theorems, where we show that these data determine a bundle up to isomorphism. The cases of the quadric hypersurface in $\mathbb{P}^{n+1}$ and the Veronese surface in $\mathbb{P}^{5}$ are considered in more detail.
\end{abstract}

\section{Introduction}

In a fundamental paper, Horrocks [1964] described all vector bundles on projective space $\mathbb{P}^{n}$ in terms of their intermediate cohomology modules. He described these cohomology modules using what he called a $\mathfrak{Z}$-complex, and showed that the category of vector bundles modulo stable equivalence was equivalent to the category of all $\mathfrak{Z}$-complexes modulo exact free complexes. In particular, this gives the well-known Horrocks criterion for a vector bundle to be a sum of line bundles in terms of the vanishing of its intermediate cohomology. His results were reformulated by Walters [1996] in the language of derived categories, and extended to sheaves by Coandă [2010]. Berlinson [1978] described the derived category of sheaves on a projective space using complexes built from an "exceptional sequence" $\left\{\mathcal{O}_{\mathbb{P}} n(-n), \ldots, \mathscr{O}_{\mathbb{P}} n(-1), \mathscr{O}_{\mathbb{P}}\right\}$ of line bundles on $\mathbb{P}^{n}$, and Kapranov [1988] gave a similar description for smooth quadric hypersurfaces by enlarging the sequence to include the spinor bundles $\Sigma$ of the quadric. Ancona and Ottaviani [1991] used these methods to extend the Horrocks splitting criterion to quadrics, with a theorem that a vector bundle $\mathscr{E}$ on a quadric $2_{n}$ (of dimension $n$ ) is a sum of line bundles if and only if $\mathscr{E}$ has its intermediate cohomology modules $H_{*}^{i}(\mathscr{E})$ all zero for $1 \leq i \leq n-1$ and also $H_{*}^{n-1}(\mathscr{E} \otimes \Sigma)=0$ for the spinor bundles $\Sigma$.

Keywords: vector bundles, cohomology modules, Horrocks correspondence, smooth ACM varieties. 
In this paper, we copy Horrocks' method on a smooth arithmetically CohenMacaulay (ACM) subvariety $X$ of projective space. Given a vector bundle $\mathscr{E}$ on $X$, we construct a $\mathfrak{Z}$-complex of free $A$-modules (where $A$ is the coordinate ring of $X$ ). This complex, when sheafified, gives rise to a vector bundle $\mathscr{F}$ on $X$ which we call a Horrocks data bundle for $\mathscr{E}$, since it comes with a map $\beta: \mathscr{F} \rightarrow \mathscr{E}$ which is an isomorphism on intermediate cohomology modules. When $H_{*}^{0}(\beta)$ is surjective, the kernel of $\beta$ is some ACM bundle on $X$.

These methods of Horrocks provide for ACM varieties a vector-bundle version of results of Auslander and Bridger [1969, Proposition 4.26, Corollary 4.27], who gave a structure theorem for a module $M$ of finite Gorenstein dimension $n$ over a commutative ring, showing that $M \oplus P$ for some projective module $P$ can be expressed as an extension of a module $H_{n}(M)$ of projective dimension $n$ by a module of zero Gorenstein dimension, where the map $M \rightarrow H_{n}(M)$ satisfies a universal property. In an unpublished preprint, Buchweitz [1986] proved a similar result for finitely generated modules over strongly Gorenstein (noncommutative) rings. We will see that the graded $A$-module $F$ of global sections of the Horrocks data bundle $\mathscr{F}$ will have $F^{\vee}$ of finite projective dimension.

With this natural extension of Horrocks' arguments to an ACM variety, we give a generalization of the Horrocks correspondence in Section 1. Our goal in looking at a Horrocks correspondence on $X$ is to look for cohomological invariants that determine $\mathscr{E}$. We will take the Horrocks data bundle as encoding all the intermediate cohomology for $\mathscr{E}$, and view it as one of the invariants. So we will study the bundles $\mathscr{E}$ with a fixed (minimal) Horrocks data bundle $\mathscr{F}$. While for the map $\mathscr{F} \rightarrow \mathscr{E}$ the induced map of first cohomology modules $H_{*}^{1}(\mathscr{F}) \rightarrow H_{*}^{1}(\mathscr{E})$ is an isomorphism, for various irreducible ACM bundles $\mathscr{B}$ on $X$, the map $H_{*}^{1}\left(\mathscr{F} \otimes \mathscr{B}^{\vee}\right) \rightarrow H_{*}^{1}\left(\mathscr{E} \otimes \mathscr{B}^{\vee}\right)$ may have a kernel. These kernels will give more cohomological invariants and we will call them modules of $\mathscr{B}$-socle elements. In Theorems 1.10 and 1.11 , we see how these invariants determine $\mathscr{E}$ up to direct sums of ACM bundles. We also give a splitting criterion for the bundle $\mathscr{E}$ to be a sum of line bundles restricted from projective space. What is lacking in Section 1 is an understanding of which modules of socle elements are obtained from a vector bundle for a general $\mathrm{ACM}$ variety.

In Section 2 we describe the case of quadrics, on which ACM bundles are well understood due to Knörrer [1987]. In particular, for the spinor bundles $\Sigma_{i}$ on a quadric $2_{n}$, modules of $\Sigma_{i}$-socle elements of a Horrocks data bundle $\mathscr{F}$ are just graded vector spaces. We show that a vector bundle $\mathscr{E}$ exists for each choice of Horrocks data bundle $\mathscr{F}$ and vector spaces $V_{i}$ of $\Sigma_{i}$-socle elements of $\mathscr{F}$, and that two vector bundles with the same data of $\mathscr{F}, V_{i}$ (up to obvious isomorphisms) are isomorphic up to direct sums of ACM bundles. In this way we generalize the results obtained in [Malaspina and Rao 2014] on $2_{2}$. 
In Section 3 we deal with the Veronese surface $\mathscr{V} \subset \mathbb{P}^{5}$. The study of vector bundles on $\mathscr{V}$ is trivial by Horrocks if we view $\mathscr{V}$ as $\mathbb{P}^{2}$. But, as another illustration of the methods, it is an interesting example of an arithmetically Cohen-Macaulay embedding which is not arithmetically Gorenstein and for which the ACM bundles are easy to handle.

\section{Horrocks data bundles on ACM varieties}

Let $X$ be a smooth ACM variety of dimension $n$ in $\mathbb{P}^{n+r}$ over a field $k$. For any sheaf $\mathscr{B}$ on $X, H_{*}^{i}(\mathscr{B})$ will denote $\bigoplus_{l \in \mathbb{Z}} H^{i}(X, \mathscr{B}(l))$. The coordinate ring of $X, A=H_{*}^{0}\left(\mathcal{O}_{X}\right)$, is a noetherian Cohen-Macaulay graded $k$-algebra. $H_{*}^{i}(\mathscr{B})$ is a graded module over $A$. Let $\mathrm{M}$ be the category of graded, finitely generated $A$-modules and graded homomorphisms. Any finitely generated projective graded $A$ module has the form $\bigoplus_{i} A\left(a_{i}\right)$ for some shifts $a_{i} \in \mathbb{Z}$ in grading, and will be called a free $A$-module. Let $\mathfrak{P} \subset \mathrm{M}$ be the full subcategory of finitely generated free $A$-modules. $\mathscr{C}^{-}(\mathrm{M})$ and $\mathscr{C}^{-}(\mathfrak{P})$ will denote the categories of all complexes, bounded above, of objects in $\mathrm{M}$ and $\mathfrak{P}$ respectively, where morphisms are maps between two complexes. Since M has enough projectives, given a complex $C^{\bullet}$ of objects in $\mathrm{M}$, bounded above, one can find a free resolution, i.e., a complex $P^{\bullet}$ in $\mathscr{C}^{-}(\mathfrak{P})$ with a quasi-isomorphism $P^{\bullet} \rightarrow C^{\bullet}$.

Let $\mathscr{E} \in \mathrm{VB}$ be an object in the category of finite-rank vector bundles on $X . H_{*}^{i}(\mathscr{E})$ is an $A$-module of finite length for $1 \leq i \leq n-1$. A vector bundle will be called free if it has the form $\bigoplus_{i} O_{X}\left(a_{i}\right)$. A vector bundle $\mathscr{E}$ will be called ACM (arithmetically Cohen-Macaulay) if $H_{*}^{i}(\mathscr{E})=0$ for all $1 \leq i \leq n-1$. Since $X$ is ACM, every free bundle is ACM. By Serre duality, the line bundle $\omega_{X}$ is an ACM line bundle.

Given $\mathscr{E}$, let $E$ denote the graded $A$-module $H_{*}^{0}(\mathscr{E})$. Denoting duals by ${ }^{\vee}$ in the categories VB and $\mathrm{M}$, we have $H_{*}^{0}(\mathscr{E} \vee) \cong\left(H_{*}^{0}(\mathscr{E})\right)^{\vee}$. Following Horrocks, we choose a resolution of $H_{*}^{0}\left(\mathscr{E}^{\vee}\right)$ by finitely generated free modules

$$
\cdots \longrightarrow C^{3 \vee} \longrightarrow C^{2 \vee} \longrightarrow C^{1 \vee} \longrightarrow C^{0 \vee} \longrightarrow H_{*}^{0}\left(\mathscr{E}^{\vee}\right) \longrightarrow 0 .
$$

In [Horrocks 1964], this could be chosen as a finite resolution, but in our case it may be infinite. However, if $K=\operatorname{ker}\left(C^{n-2 \vee} \rightarrow C^{n-3 \vee}\right)$, then $\mathscr{K}$ is an ACM vector bundle on $X$, where $\mathscr{K}=\widetilde{K}$ is the sheaf obtained from $K$. Replacing the terms up to and including $C^{n-1 \vee}$ by $K$ and dualizing, we get the complex

$$
C_{\{0, n\}}^{\bullet}: 0 \longrightarrow C^{0} \stackrel{\delta_{C}^{1} \bullet}{\longrightarrow} C^{1} \stackrel{\delta_{C}^{2} \bullet}{\longrightarrow} C^{2} \stackrel{\delta_{C}^{3} \bullet}{\longrightarrow} \cdots \stackrel{\delta_{C^{\bullet}}^{n-2}}{\longrightarrow} C^{n-2} \longrightarrow K^{\vee} \longrightarrow 0 .
$$

The exact sequence (1), when sheafified, gives an exact sequence of vector bundles, and its dual gives the exact sequence of vector bundles

$$
0 \longrightarrow \mathscr{E} \longrightarrow \widetilde{C}^{0} \stackrel{\delta_{C}^{1} \bullet}{\longrightarrow} \widetilde{C}^{1} \stackrel{\delta_{C}^{2} \bullet}{\longrightarrow} \widetilde{C}^{2} \stackrel{\delta_{C}^{3} \bullet}{\longrightarrow} \cdots \stackrel{\delta_{C}^{n-2}}{\longrightarrow} \widetilde{C}^{n-2} \longrightarrow \mathcal{K}^{\vee} \longrightarrow 0 .
$$


From this it becomes evident that $E=H_{*}^{0}(\mathscr{\mathscr { C }})$ is given as $H^{0}\left(C_{\{0, n\}}^{\bullet}\right)$, and $H_{*}^{i}(\mathscr{E})=H^{i}\left(C_{\{0, n\}}^{\bullet}\right)$ for $i=1, \ldots n-1$ (where $C_{\{0, n\}}^{n-1}$ is understood to refer to $K^{\vee}$ ).

$E$ itself has a free resolution (again possibly infinite). Splice $C_{\{0, n\}}^{\bullet}$ with a free resolution $L^{\bullet}$ of $E$ and call the resulting complex $C^{\bullet}$. The complex $C^{\bullet}$ is bounded above and has the property that $H^{i}\left(C^{\bullet}\right)=H_{*}^{i}(\mathscr{E})$ for $i=1, \ldots n-1$ and equals 0 for other values of $i$.

Choose a free resolution $P^{\bullet}$ in $\mathscr{C}^{-}(\mathfrak{P})$ of $C^{\bullet}$ :

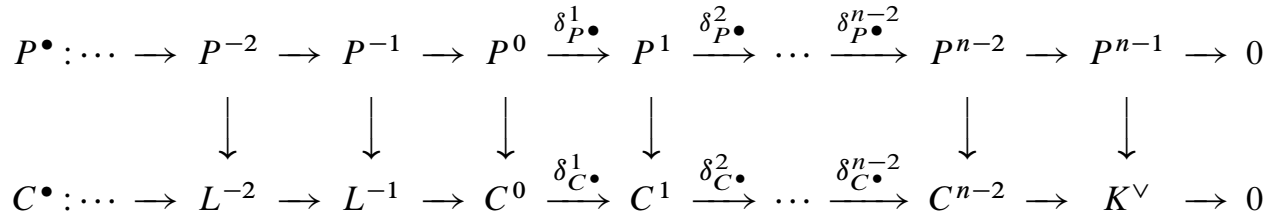

Then $P^{\bullet}$ is an element in $\mathscr{C}^{-}(\mathfrak{P})$ with the property that $H^{i}\left(P^{\bullet}\right)$ is an $A$-module of at most finite length for $1 \leq i \leq n-1$ and is zero for other $i$. In [Horrocks 1964] the bounded version of such a free complex was called a $\mathfrak{Z}$-complex, while Walters [1996] called the category of such complexes FinL(P). In our setting, we will call it a Horrocks data complex and use the notation of [Walter 1996]. We also define a "Horrocks data bundle" for each such Horrocks data complex:

Definition 1.1. FinL ${ }^{-}(\mathfrak{P})$ is the full subcategory of all complexes $P^{\bullet}$ in $\mathscr{C}^{-}(\mathfrak{P})$ with the property that $H^{i}\left(P^{\bullet}\right)$ is an $A$-module of at most finite length for $1 \leq i \leq n-1$ and is zero for other $i$. A complex $P^{\bullet}$ in FinL ${ }^{-}(\mathfrak{P})$ will be called a Horrocks data complex. For such a complex, let $F=\operatorname{ker}\left(\delta_{P \bullet}^{1}: P^{0} \rightarrow P^{1}\right)$. Then the sheaf $\mathscr{F}=\tilde{F}$ will be called a Horrocks data bundle on $X$.

It should be clear that the above $\mathscr{F}$ is a vector bundle on $X$ with the property that $H_{*}^{i}(\mathscr{F})=H^{i}\left(P^{\bullet}\right)$ for $1 \leq i \leq n-1$.

Lemma 1.2 [Horrocks 1964, Theorem 7.2]. $F^{\vee}$ has a finite free resolution.

Proof. Horrocks' proof cited above is when $A$ is a regular ring, but remains valid when $A$ is Cohen-Macaulay. Another proof (indicated by the referee) is: $0 \rightarrow\left(P^{n-1}\right)^{\vee} \rightarrow\left(P^{n-2}\right)^{\vee} \rightarrow \cdots \rightarrow\left(P^{0}\right)^{\vee} \rightarrow F^{\vee} \rightarrow 0$ is a complex in M, locally free and exact away from the maximal ideal for the vertex of the cone over $X$, and hence is exact by the Peskine-Szpiro acyclicity lemma.

Since the modules of global sections of a nonfree ACM bundle and of its dual bundle on $X$ have infinite projective dimension over $A$, it follows that a Horrocks data bundle $\mathscr{F}$ can have no nonfree ACM bundle or its dual as a summand.

Since any $P^{\bullet}$ in $\mathscr{C}^{-}(\mathfrak{P})$ decomposes as $M^{\bullet} \oplus L^{\bullet}$, where $M^{\bullet}$ is a minimal free complex and $L^{\bullet}$ is an acyclic free complex, we get $\mathscr{F}=\mathscr{F}_{\min } \oplus \mathscr{L}$, where $\mathscr{F}_{\text {, }} \mathscr{F}_{\text {min }}$, and $\mathscr{L}$ are the Horrocks data bundles corresponding to $P^{\bullet}, M^{\bullet}$, and $L^{\bullet}$ respectively. 
$\mathscr{L}$ is a free bundle and $\mathscr{F}_{\min }$ will be called a "minimal" Horrocks data bundle. The projective space version of the following isomorphism theorem can be found in [Horrocks 1964, Theorem 7.5, Proposition 9.5] or [Walter 1996, Lemma 2.11].

Proposition 1.3. Let $\sigma: \mathscr{F}^{\rightarrow} \rightarrow \mathscr{F}^{\prime}$ be a homomorphism between two minimal Horrocks data bundles on $X$ such that $\sigma$ induces isomorphisms $H_{*}^{i}\left(\mathscr{F}^{\prime}\right) \rightarrow H_{*}^{i}\left(\mathscr{F}^{\prime}\right)$ for $1 \leq i \leq n-1$. Then $\sigma$ is an isomorphism.

Proof. The proofs of the results cited above work in our ACM setting as well.

Returning to the vector bundle $\mathscr{E}$, let $P^{\bullet}$ be a free resolution of $C^{\bullet}$ as described above. Let $P_{\geq 0}^{\bullet}$ denote the naive truncation of $P^{\bullet}$ at the zeroth term. We get the induced homomorphism of complexes

$$
P_{\geq 0}^{\bullet} \rightarrow C_{\{0, n\}}^{\bullet} .
$$

For $F$ defined as $\operatorname{ker} \delta_{P \bullet}^{1}$, there is an induced homomorphism $F \rightarrow E$. For the Horrocks data bundle $\mathscr{F}=\widetilde{F}$, we get a homomorphism $\beta: \mathscr{F} \rightarrow \mathscr{E}$ which induces isomorphisms $H_{*}^{i}(\mathscr{F}) \rightarrow H_{*}^{i}(\mathscr{E})$ for $1 \leq i \leq n-1$. Hence any vector bundle $\mathscr{E}$ has a "Horrocks datum", as we now define:

Definition 1.4. Let $\mathscr{E}$ be a vector bundle on $X$. A pair $(\mathscr{F}, \beta)$ will be called a Horrocks datum for $\mathscr{E}$ if $\mathscr{F}$ is a Horrocks data bundle and $\beta$ is a homomorphism $\beta: \mathscr{F} \rightarrow \mathscr{E}$ which induces isomorphisms $H_{*}^{i}(\mathscr{F}) \rightarrow H_{*}^{i}(\mathscr{E})$ for $1 \leq i \leq n-1$.

A point on terminology: Auslander's approximation theorem [Auslander and Bridger 1969, Proposition 4.26, Corollary 4.27] quoted in the introduction states that, given a module $M$ of finite Gorenstein dimension $n$, there exist a projective module $P$, a module $H_{n}(M)$ of projective dimension $n$, a module $M_{n}$ of zero Gorenstein dimension and an exact sequence $0 \rightarrow M_{n} \rightarrow M \oplus P \rightarrow H_{n}(M) \rightarrow 0$. Following Auslander's suggestion, Buchweitz [1986, Corollary 5.3.3] called $H_{n}(M)$ (with the map $M \rightarrow H_{n}(M)$ ) a "hull of finite projective dimension" for $M$, and $M_{n}$ the maximal Cohen-Macaulay approximation to $M$.

In the case where our variety $X$ is arithmetically Gorenstein, Auslander's sequence can be seen as coming from the dual of the $\eta$-sequence of Theorem 1.7 below: given $E$, the $\eta$-sequence $0 \rightarrow K \rightarrow F \rightarrow E \rightarrow 0$ dualized gives $0 \rightarrow E^{\vee} \rightarrow F^{\vee} \rightarrow K^{\vee}$, where $F^{\vee}$ has finite projective dimension. When $X$ is arithmetically Gorenstein, $K^{\vee}$ is a maximal Cohen-Macaulay module and $F^{\vee} \rightarrow K^{\vee}$ is surjective. Pull back the exact sequence by a surjection $L \rightarrow K^{\vee} \rightarrow 0$, with $L$ projective. It splits. This induces an exact sequence $0 \rightarrow N \rightarrow E^{\vee} \oplus L \rightarrow F^{\vee} \rightarrow 0$, where $N$ (the kernel of $L \rightarrow K^{\vee}$ ) is a maximal Cohen-Macaulay module. This fits the above approximation theorem for $E^{\vee}$. 
However, we have chosen the notation "FF is the Horrocks data bundle for $\mathscr{E}$ " since $\mathscr{F}$ encodes all the intermediate cohomology data of $\mathscr{E}$.

Theorem 1.5. Let $\mathscr{E}_{1}, \mathscr{E}_{2}$ be vector bundles on $X$ with Horrocks data $\left(\mathscr{F}_{1}, \beta_{1}\right)$, $\left(\mathscr{F}_{2}, \beta_{2}\right)$ respectively. Let $\sigma: \mathscr{E}_{1} \rightarrow \mathscr{E}_{2}$ be a homomorphism.

(1) There is a free bundle $\mathscr{E}$ and a commuting square

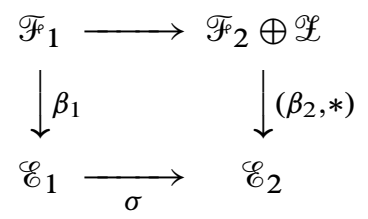

(2) If $H_{*}^{0}\left(\beta_{2}\right): H_{*}^{0}\left(\mathscr{F}_{2}\right) \rightarrow H_{*}^{0}\left(\mathscr{E}_{2}\right)$ is surjective, the free bundle $\mathscr{E}$ can be chosen to be zero.

Proof. It is straightforward to see that the construction of the complex $C^{\bullet}$ out of the vector bundle $\mathscr{E}$ is functorial in the sense that, given $\sigma: \mathscr{E}_{1} \rightarrow \mathscr{E}_{2}$, there is an induced morphism from $C_{1}^{\bullet} \rightarrow C_{2}^{\bullet}$ with the property that the homomorphisms $H^{i}\left(C_{1}^{\bullet}\right) \rightarrow H^{i}\left(C_{2}^{\bullet}\right)$ coincide with $H^{i}(\sigma): H_{*}^{i}\left(\mathscr{E}_{1}\right) \rightarrow H_{*}^{i}\left(\mathscr{E}_{2}\right)$ for $1 \leq i \leq n-1$. In the special case of $\beta_{k}: \mathscr{F}_{k} \rightarrow \mathscr{E}_{k}$, a Horrocks datum, we get a quasi-isomorphism $P_{k}^{\bullet} \rightarrow \mathscr{C}_{k}^{\bullet}$, where $P_{k}^{\bullet}$ is the Horrocks data complex associated to $\mathscr{F}_{k}$, so that $P_{k}^{\bullet} \rightarrow \mathscr{C}_{k}^{\bullet}$ is a free resolution of $\mathscr{C}_{k}^{\bullet}$. Now given a morphism of complexes $C_{1}^{\bullet} \rightarrow C_{2}^{\bullet}$, we can lift the morphism to their free resolutions, after adding a free acyclic complex to $P_{2}^{\bullet}$. This gives the commuting square of part (1). The proof of part (2) is elementary.

The following theorems (Theorems 1.6 and 1.7) are to be found in more general form in [Buchweitz 1986] as the "syzygy theorem for Gorenstein rings". The diagram in Theorem 1.8 below is Buchweitz's octahedron [1986, (5.3.1)].

Theorem 1.6 ( $\gamma$ sequence for $\mathscr{E})$. Let $\mathscr{E}$ be a vector bundle on $X$ and $(\mathscr{F}, \beta)$ a Horrocks datum for $\mathscr{E}$. From the Horrocks data complex $P^{\bullet}$ for $\mathscr{F}$, consider the exact sequence $\Psi: 0 \rightarrow \mathscr{F} \rightarrow \mathscr{P}^{0} \rightarrow \mathscr{G} \rightarrow 0$, where $\mathscr{P}^{0}=\widetilde{P}^{0}$ and $\mathscr{G}=\widetilde{G}$ with $G=\operatorname{ker} \delta_{P}^{2}$. We define $\gamma$ as the pushout of $\Psi$ by $\beta$

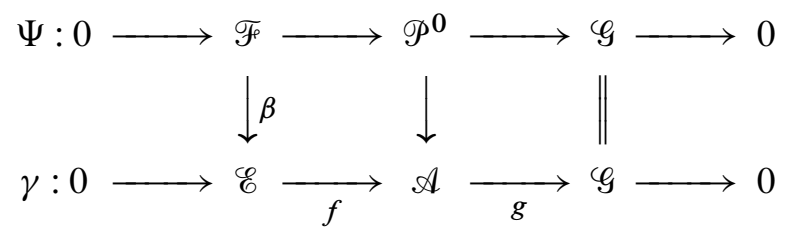

(1) Given two bundles $\mathscr{E}_{1}, \mathscr{E}_{2}$, a morphism $\sigma: \mathscr{E}_{1} \rightarrow \mathscr{E}_{2}$, and Horrocks data $\left(\mathscr{F}_{1}, \beta_{1}\right),\left(\mathscr{F}_{2}, \beta_{2}\right)$ for each bundle, we obtain a commuting box of short exact 
sequences (using obvious notation)

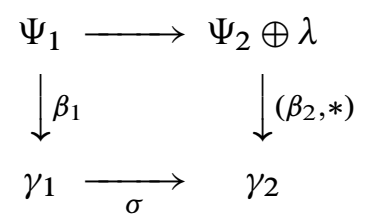

where $\lambda$ is a short exact sequence $0 \rightarrow \mathscr{L} \rightarrow \mathscr{L} \rightarrow 0 \rightarrow 0$ of free bundles. If $H_{*}^{0}\left(\beta_{2}\right)$ is surjective onto $H_{*}^{0}\left(\mathscr{E}_{2}\right), \lambda$ may be taken to be zero.

(2) $H_{*}^{n-1}(\mathscr{G})=0$, and $\mathscr{A}$ is an ACM bundle on $X$.

(3) Up to a short exact sequence $0 \rightarrow 0 \rightarrow \mathscr{L} \rightarrow \mathscr{L} \rightarrow 0$ of free bundles, the sequence $\gamma$ depends only on $\mathscr{E}$ and not on the choice of Horrocks datum.

Proof. (1) $\sigma$ lifts to a map $\mathscr{F}_{1} \rightarrow \mathscr{F}_{2} \oplus \mathscr{E}$ to give a commuting square, by Theorem 1.5. $\mathscr{F}_{2} \oplus \mathscr{L}$ is a Horrocks data bundle for the Horrocks data complex, where $P^{0}$ is replaced by $P^{0} \oplus \mathscr{E}$ but with the same bundle $\mathscr{G}_{2}$. It is easy to see that the map $\mathscr{F}_{1} \rightarrow \mathscr{F}_{2} \oplus \mathscr{L}$ extends to a map of sequences $\Psi_{1} \rightarrow \Psi_{2} \oplus \lambda$. The pushouts of $\Psi_{2}$ and $\Psi_{2} \oplus \lambda$ give the same sequence $\gamma_{2}$. Lastly, since we have a commuting square from the first line of the proof, the pushouts of $\Psi_{1}$ and $\Psi_{2} \oplus \lambda$ give a commuting box of exact sequences.

(2) By construction, $H_{*}^{n-1}(\varphi)=H^{n}\left(P^{\bullet}\right)=0$. Since we have isomorphisms $H_{*}^{i}(\mathscr{G}) \cong H_{*}^{i+1}(\mathscr{F}) \cong H_{*}^{i+1}(\mathscr{E})$ for $1 \leq i \leq n-2$ and $H_{*}^{0}(\mathscr{G}) \rightarrow H_{*}^{1}(\mathscr{F}) \cong H_{*}^{1}(\mathscr{E})$, we conclude that $\mathscr{A}$ is ACM.

(3) This follows from the first part when we apply the previous theorem to the identity morphism from $\mathscr{E}$ to $\mathscr{E}$. Indeed, the theorem, together with Proposition 1.3, shows that any two Horrocks data bundles for $\mathscr{E}$ are stably equivalent.

Theorem 1.7 ( $\eta$ sequence for $\mathscr{E})$. Let $(\mathscr{F}, \beta)$ be a Horrocks datum for the bundle $\mathscr{E}$ such that $H_{*}^{0}(\beta)$ is surjective. We define the $\eta$ sequence for $\mathscr{E}$ to be

$$
0 \longrightarrow \mathscr{K} \longrightarrow \mathscr{F} \stackrel{\beta}{\longrightarrow} \mathscr{E} \longrightarrow 0
$$

where $\mathscr{K}$ is the kernel bundle.

(1) $\mathscr{Y}$ is an ACM bundle.

(2) $\eta$ is determined by $\mathscr{E}$ up to a short exact sequence $0 \rightarrow \mathscr{L} \rightarrow \mathscr{L} \rightarrow 0 \rightarrow 0$ of free bundles.

(3) Given a morphism $\sigma: \mathscr{E}_{1} \rightarrow \mathscr{E}_{2}$, there is an induced morphism of short exact sequences $\eta_{1} \rightarrow \eta_{2}$. 
Proof. The proof is easy. We just mention that the induced map $\eta_{1} \rightarrow \eta_{2}$ depends on the choice of a map from $\mathscr{F}_{1}$ to $\mathscr{F}_{2}$ that lifts $\sigma$ (as obtained from Theorem 1.5).

Theorem 1.8 (diagram of $\mathscr{E})$. Let $(\mathscr{F}, \beta)$ be a Horrocks datum for the bundle $\mathscr{E}$ such that $H_{*}^{0}(\beta)$ is surjective. The $\gamma$ and $\eta$ sequences of $\mathscr{E}$ fit into a diagram for $\mathscr{E}$

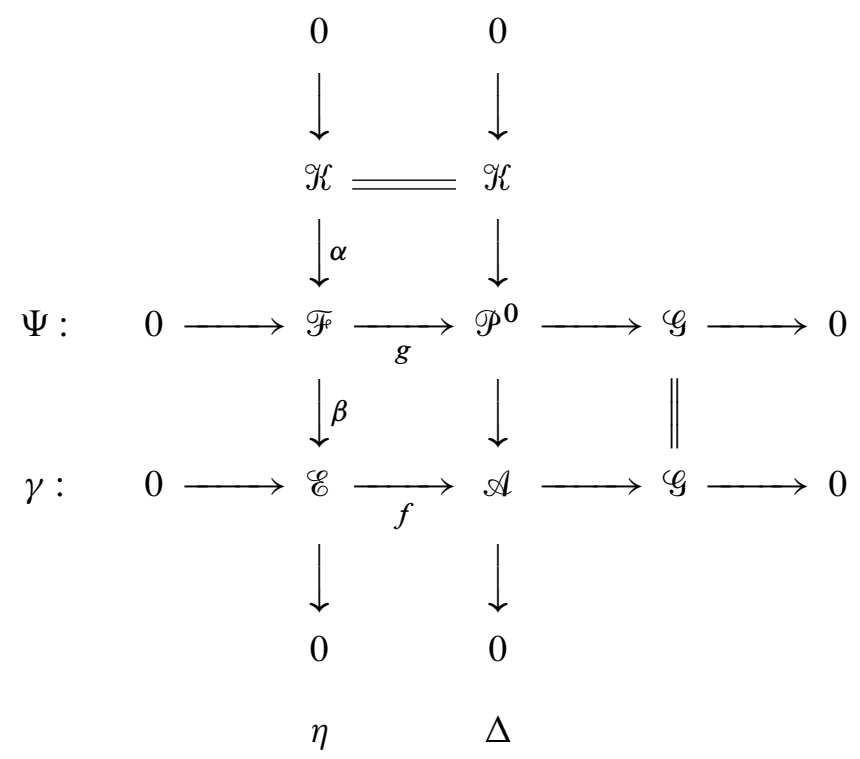

Given a morphism $\sigma: \mathscr{E}_{1} \rightarrow \mathscr{E}_{2}$, there is an induced map from the diagram of $\mathscr{E}_{1}$ to the diagram of $\mathscr{E}_{2}$.

Proof. While the existence of the diagram is clear, the map from diagram of $\mathscr{E}_{1}$ to the diagram of $\mathscr{E}_{2}$ with appropriate commuting boxes exists because the choice of a map from $\mathscr{F}_{1}$ to $\mathscr{F}_{2}$ that lifts $\sigma$ will determine $\eta_{1} \rightarrow \eta_{2}$ and then allows a choice of a map $\Psi_{1} \rightarrow \Psi_{2}$. This now gives the commuting box of short exact sequences of Theorem 1.6.

The following is a criterion for obtaining a map between two $\gamma$-sequences:

Proposition 1.9. Let $\mathscr{C}^{\mathscr{E}}, \mathscr{C}^{\prime}$ be two vector bundles with the same (minimal) Horrocks data bundle $\mathscr{F}_{\text {min }}$ and Horrocks data $\left(\mathscr{F}_{\text {min }}, \beta\right),\left(\mathscr{F}_{\text {min }}, \beta^{\prime}\right)$. Let $\mathscr{\Re}_{1}, \mathscr{P}_{2}, \ldots, \mathscr{B}_{k}$ be the distinct nonfree irreducible ACM bundles (up to twists by $\mathrm{O}_{X}(a)$ ) that appear as summands in the middle term $A_{\mathscr{E}}$ of the $\gamma$-sequence of $\mathscr{E}$. For each $\mathscr{B}_{i}$, let $V_{i}$ be the kernel of the map $H_{*}^{1}\left(\beta \otimes 1_{\mathscr{B}_{i}^{\vee}}\right)$ from $H_{*}^{1}\left(\mathscr{F}_{\min } \otimes \mathscr{B}_{i}^{\vee}\right)$ to $H_{*}^{1}\left(\mathscr{E} \otimes \mathscr{B}_{i}^{\vee}\right)$, and let $V_{i}^{\prime}$ be the same with $\beta$ replaced by $\beta^{\prime}$. If $V_{i} \subseteq V_{i}^{\prime}$ for all $i$, then there exists a map $\phi: \mathscr{E} \rightarrow \mathscr{E}^{\prime}$ such that the $\gamma$-sequence of $\mathscr{E}^{\prime}$ is the pushout by $\phi$ of the $\gamma$-sequence for $\mathscr{E}$. 
Proof. Since the $\gamma$-sequences $\gamma, \gamma^{\prime}$ are pushouts by $\beta, \beta^{\prime}$ of the $\Psi$-sequence for $\mathscr{F}_{\text {min }}$

$$
\Psi: 0 \longrightarrow \mathscr{F}_{\min } \longrightarrow \mathscr{P}^{0} \longrightarrow \mathscr{G}_{\min } \longrightarrow 0
$$

in the commutative diagram

$$
\begin{gathered}
\operatorname{Hom}\left(\mathscr{P}^{0}, \mathscr{E}^{\prime}\right) \longrightarrow \operatorname{Hom}\left(\mathscr{F}_{\min }, \mathscr{E}^{\prime}\right) \underset{\delta(\Psi)}{\longrightarrow} \operatorname{Ext}^{1}\left(\mathscr{G}_{\min }, \mathscr{E}^{\prime}\right) \longrightarrow \operatorname{Ext}^{1}\left(\mathscr{P}^{0}, \mathscr{E}^{\prime}\right) \\
\uparrow_{\beta} \\
\operatorname{Hom}\left(\mathscr{E}, \mathscr{E}^{\prime}\right) \underset{\delta(\gamma)}{\longrightarrow} \operatorname{Ext}^{1}\left(\mathscr{G}_{\min }, \mathscr{E}^{\prime}\right) \longrightarrow \operatorname{Ext}^{1}\left(\mathscr{A}_{\mathscr{E}}, \mathscr{E}^{\prime}\right)
\end{gathered}
$$

it suffices to show that $\gamma^{\prime} \in \operatorname{Ext}^{1}\left(\mathscr{G}_{\text {min }}, \mathscr{E}^{\prime}\right)$ maps to zero in $\operatorname{Ext}^{1}\left(\mathscr{A}_{\mathscr{E}}, \mathscr{E}^{\prime}\right)$, for then there is an element $\sigma \in \operatorname{Hom}\left(\mathscr{E}, \mathscr{C}^{\prime}\right)$ such that $\sigma \circ \beta$ differs from $\beta^{\prime}$ by a map that factors through $\mathscr{P}^{0}$.

Let $\rho: \mathscr{A}_{\mathscr{E}} \rightarrow \mathscr{G}_{\min }$ be the map occurring in the $\gamma$-sequence of $\mathscr{E}$. Then under the connecting homomorphism for $\gamma \otimes \mathscr{A}_{\mathscr{E}}^{\vee}, \rho$ maps to zero under $H_{*}^{0}\left(\varphi_{\min } \otimes \mathscr{A}_{\mathscr{E}}^{\vee}\right) \rightarrow$ $H_{*}^{1}\left(\mathscr{E} \otimes \mathscr{A}_{\mathscr{E}}^{\vee}\right)$. Hence, under the connecting homomorphism of $\Psi \otimes A_{\mathscr{E}}^{\vee}, \rho$ maps to the kernel of $H_{*}^{1}\left(\mathscr{F}_{\min } \otimes \mathscr{A}_{\mathscr{E}}^{\vee}\right) \rightarrow H_{*}^{1}\left(\mathscr{E} \otimes \mathscr{A}_{\mathscr{E}}^{\vee}\right)$. By the assumption $V_{i} \subseteq V_{i}^{\prime}$ for all $i, \rho$ also maps to the kernel of $H_{*}^{1}\left(\mathscr{F}_{\min } \otimes \mathscr{A}_{\mathscr{E}}^{\vee}\right) \rightarrow H_{*}^{1}\left(\mathscr{E}^{\prime} \otimes \mathscr{A}_{\mathscr{E}}^{\vee}\right)$. It follows that the pullback of $\gamma^{\prime}$ by $\rho$ splits, which was the desired result.

This criterion leads to an isomorphism theorem on $X$ :

Theorem 1.10 (isomorphism theorem). Let $\mathscr{E}, \mathscr{E}$ ' be two vector bundles on $X$, with the same minimal Horrocks data bundle $\mathscr{F}_{\min }$ and Horrocks data $\left(\mathscr{F}_{\min }, \beta\right)$, $\left(\mathscr{F}_{\min }, \beta^{\prime}\right)$. Let $\mathscr{B}_{1}, \mathscr{B}_{2}, \ldots, \mathscr{B}_{k}$ be the distinct nonfree irreducible ACM bundles (up to twists by $\mathrm{O}_{X}(a)$ ) that appear as summands in either of the middle terms $A_{\mathscr{E}}, A_{\mathscr{E}}$ of the $\gamma$-sequences of $\mathscr{E}, \mathscr{E}^{\prime}$. If for each $i$ the kernel of $H_{*}^{1}\left(\beta \otimes 1_{\mathscr{B}_{i}}\right)$ equals the kernel of $H_{*}^{1}\left(\beta^{\prime} \otimes 1_{\mathscr{B}_{i}^{\vee}}\right)$ and if $\mathscr{E}$ and $\mathscr{E}^{\prime}$ have no ACM summands, then $\mathscr{E} \cong \mathscr{E}$.

Proof. If $\mathscr{F}$ is free, $\mathscr{E}, \mathscr{E}^{\prime}$ are ACM and the theorem does not apply. So we will assume that $\mathscr{F}_{\min }$ is a nonfree minimal Horrocks data bundle. By applying Proposition 1.9, there exists a homomorphism $\sigma: \mathscr{E} \rightarrow \mathscr{E}^{\prime}$ and a commutative diagram of $\gamma$-sequences

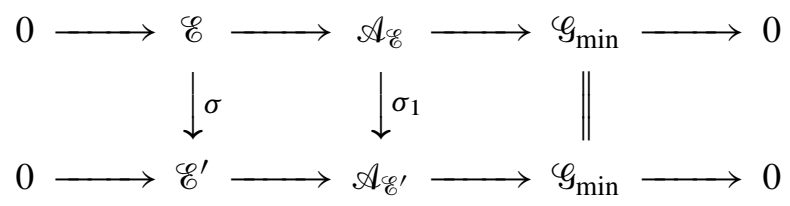

Tensor the diagram by $\mathscr{B}^{\vee}$, where $\mathscr{B}$ will stand for any of the distinct irreducible ACM bundles (up to twists by $\mathscr{O}_{X}(a)$ ) that appear as summands in $\mathscr{A}_{\mathscr{E}}$, including 
the possible free line bundle $\mathrm{O}_{X}$. In the induced diagram of cohomology, we get

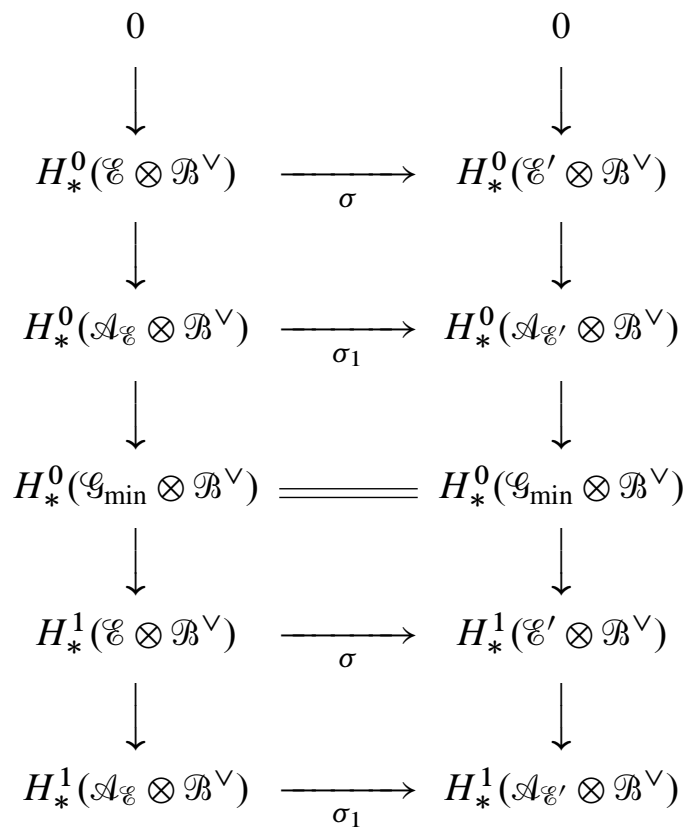

The map $H_{*}^{0}\left(\mathscr{G}_{\min } \otimes \mathscr{B}^{\vee}\right) \rightarrow H_{*}^{1}\left(\mathscr{E} \otimes \mathscr{B}^{\vee}\right)$ factors through $H_{*}^{1}\left(\mathscr{F} \otimes \mathscr{B}^{\vee}\right)$, since $\gamma$ is the pushout of $\Psi$ by $\beta$. The condition of equality of kernels for $H_{*}^{1}\left(\beta \otimes 1_{\mathscr{R}} \vee\right)$ and $H_{*}^{1}\left(\beta^{\prime} \otimes 1_{\mathscr{B}} \vee\right)$ implies that the kernel in $H_{*}^{0}\left(\mathscr{G}_{\min } \otimes \mathscr{B}^{\vee}\right)$ is the same for $\mathscr{E}$ and $\mathscr{E}^{\prime}$. Therefore the mapping cone map $H_{*}^{0}\left(\mathscr{E}^{\prime} \otimes \mathscr{B}^{\vee}\right) \oplus H_{*}^{0}\left(\mathscr{A}_{\mathscr{E}} \otimes \mathscr{B}^{\vee}\right) \rightarrow H_{*}^{0}\left(\mathscr{A}_{\mathscr{E}} \otimes^{\prime} \otimes \mathscr{B}^{\vee}\right)$ is surjective. Viewing each summand $\mathscr{B}$ of $\mathscr{A}_{\mathscr{E}}$, the identity global section in $H^{0}\left(\mathscr{B} \otimes \mathscr{B}^{\vee}\right)$ is in the image of this surjection. It cannot be in the image of $H_{*}^{0}\left(\mathscr{E}^{\prime} \otimes \mathscr{B}^{\vee}\right)$ since $\mathscr{E}^{\prime}$ does not have $\mathscr{B}$ as a summand. Hence it is in the image of some $\mathscr{B}^{\prime}$ term in $\mathscr{A}_{\mathscr{E}}$. This forces $\mathscr{B}^{\prime}$ to equal $\mathscr{B}$, and the map $\sigma_{1}: \mathscr{A}_{\mathscr{E}} \rightarrow \mathscr{A}_{\mathscr{E}}$ has to split over this $\mathscr{B}$ term in $\mathscr{A}_{\mathscr{E}}$.

It follows that $\sigma_{1}$ is a (split) surjection. Hence $\sigma: \mathscr{E} \rightarrow \mathscr{E}^{\prime}$ is onto. The roles of $\mathscr{E}, \mathscr{E}^{\prime}$ can be interchanged, showing that they are bundles of the same rank. Hence $\sigma: \mathscr{E} \cong \mathscr{E}^{\prime}$

The following theorem is in the same vein, and extends Proposition 1.3:

Theorem 1.11. Let $\sigma: \mathscr{E} \rightarrow \mathscr{E}^{\prime}$ be a sheaf homomorphism between two vector bundles on $X$, where $\mathscr{E}^{\prime}$ has no ACM summands. Suppose that $\sigma$ induces isomorphisms $H_{*}^{i}(\mathscr{E}) \rightarrow H_{*}^{i}\left(\mathscr{E}^{\prime}\right)$ for $1 \leq i \leq n-1$, and also, for each nonfree irreducible ACM bundle $\mathscr{B}$ appearing in $\mathscr{A}_{\mathscr{E}}$, suppose that the induced map $H_{*}^{1}\left(\mathscr{E} \otimes \mathscr{B}^{\vee}\right) \rightarrow H_{*}^{1}\left(\mathscr{E}^{\prime} \otimes \mathscr{B}^{\vee}\right)$ is an isomorphism. Then $\sigma$ is a split surjection decomposing $\mathscr{E}$ into $\mathscr{E}^{\prime} \oplus \mathscr{C}$, where $\mathscr{C}$ is an ACM bundle. 
Proof. By Theorem 1.5, $\sigma$ can be lifted to a map $\tilde{\sigma}: \mathscr{F}_{\text {min }} \rightarrow \mathscr{F}_{\min }^{\prime}$ of minimal Horrocks data bundles. Since $H_{*}^{i}(\tilde{\sigma})$ is an isomorphism for $1 \leq i \leq n-1, \tilde{\sigma}$ is an isomorphism. So, for convenience, we may assume that $\mathscr{F}_{\min }=\mathscr{F}_{\min }^{\prime}$, and, according to Theorem 1.6, $\sigma$ induces a map of $\gamma$-sequences

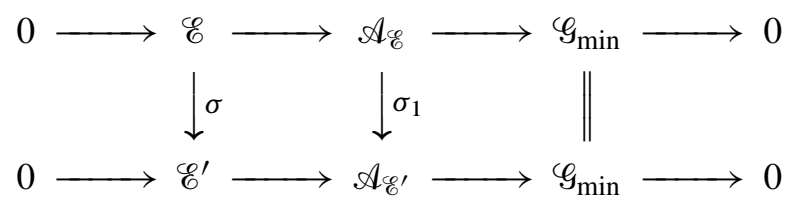

For each $\mathscr{B}$ appearing in $\mathscr{A}_{\mathscr{E}}$, as in the proof of the previous theorem after tensoring by $\mathscr{B}^{\vee}$ we can look at the diagram of cohomology. Since $H_{*}^{1}\left(\mathscr{E} \otimes \mathscr{B}^{\vee}\right) \rightarrow$ $H_{*}^{1}\left(\mathscr{E}^{\prime} \otimes \mathscr{B}^{\vee}\right)$ is an isomorphism, the kernel in $H_{*}^{0}\left(\mathscr{G}_{\min } \otimes \mathscr{B}^{\vee}\right)$ is the same for $\mathscr{E}$ and $\mathscr{E}^{\prime}$. The previous argument repeats to show that the homomorphism $\sigma_{1}: \mathscr{A}_{\mathscr{E}} \rightarrow \mathscr{A}_{\mathscr{E} \prime}$ is a split surjection, with a kernel $\mathscr{C}$ which is ACM. Hence $\sigma: \mathscr{E} \rightarrow \mathscr{\mathscr { E }}$ is also a split surjection with kernel equal to $\mathscr{C}$.

Since the $A$-submodules $V_{i}=\operatorname{ker}\left(H_{*}^{1}\left(\mathscr{F}_{\min } \otimes \mathscr{S}_{i}^{\vee}\right) \rightarrow H_{*}^{1}\left(\mathscr{E} \otimes \mathscr{B}_{i}^{\vee}\right)\right)$ play such an important role in the above description of a bundle $\mathscr{E}$, it is worthwhile to make the following definition describing its properties:

Definition 1.12. Let $\mathscr{F}$ be a sheaf on $X$ and $\mathscr{B}$ an ACM bundle on $X$ with a minimal set of generators for $H_{*}^{0}(\mathscr{B})$ given by $\bigoplus_{j} \mathcal{O}_{X}\left(a_{j}\right) \rightarrow \mathscr{B} \rightarrow 0$. The kernel of $H_{*}^{1}\left(\mathscr{F}_{\mathcal{B}} \otimes \mathscr{P}^{\vee}\right) \rightarrow H_{*}^{1}\left(\mathscr{F} \otimes \bigoplus_{j} \mathcal{O}_{X}\left(-a_{j}\right)\right)$ will be called the $A$-module of $\mathscr{P}_{B}$-socle elements for $\mathscr{F}$ and denoted by $H_{*}^{1}\left(\mathscr{F} \otimes \mathscr{B}^{\vee}\right)_{\text {soc }}$. A homogeneous element in this kernel in degree $d$ will be a $\mathscr{B}$-socle element in $H^{1}\left(\mathscr{F}(d) \otimes \mathscr{B}^{\vee}\right)$.

Remark 1.13. (1) For a vector bundle $\mathscr{F}$, the module of $\mathscr{B}$-socle elements for $\mathscr{F}$ has finite length over the field $k$.

(2) Suppose $\mathscr{B}^{\vee} \rightarrow \mathscr{O}_{X}(b)$ is any map. Then, for any sheaf $\mathscr{F}$, a $\mathscr{B}$-socle element in $H_{*}^{1}\left(\mathscr{F} \otimes \mathscr{B}^{\vee}\right)$ maps to zero in $H_{*}^{1}(\mathscr{F}(b))$, since $\mathscr{B}^{\vee} \rightarrow \mathcal{O}_{X}(b)$ factors through $\bigoplus_{j} \mathrm{O}_{X}\left(-a_{j}\right)$.

(3) Suppose $\mathscr{E}$ is a bundle on $X$ with Horrocks datum $\left(\mathscr{F}_{\min }, \beta\right)$. Then, for any ACM bundle $\mathscr{B}$, the module $V=\operatorname{ker}\left(H_{*}^{1}\left(\mathscr{F}_{\min } \otimes \mathscr{B}^{\vee}\right) \rightarrow H_{*}^{1}\left(\mathscr{E} \otimes \mathscr{B}^{\vee}\right)\right)$ consists of $\mathscr{P}_{B}$-socle elements for $\mathscr{F}_{\text {min }}$. Indeed, the map $H_{*}^{1}\left(\mathscr{F}_{\min } \otimes \bigoplus_{j} \mathscr{O}_{X}\left(-a_{j}\right)\right) \rightarrow$ $H_{*}^{1}\left(\mathscr{E} \otimes \bigoplus_{j} \mathcal{O}_{X}\left(-a_{j}\right)\right)$ is an isomorphism.

Example 1.14. Any ACM variety $X$ with a nondegenerate embedding into $\mathbb{P}^{N}$ has a Horrocks data bundle given by $\left.\Omega_{\mathbb{P}}^{1}\right|_{X}$ with $H_{*}^{1}\left(\left.\Omega_{\mathbb{P}}^{1}\right|_{X}\right)=k$ and with an exact sequence

$$
\left.0 \longrightarrow \Omega_{\mathbb{P}}^{1}\right|_{X} \longrightarrow \mathrm{O}_{X}(-1)^{\oplus N+1} \longrightarrow \mathrm{O}_{X} \longrightarrow 0 .
$$


For any ACM bundle $\mathscr{B}$ on $X$, without free summands and with $\mathscr{B}^{\vee} \hookrightarrow \bigoplus_{j} \mathrm{O}_{X}\left(-a_{j}\right)$, consider the diagram

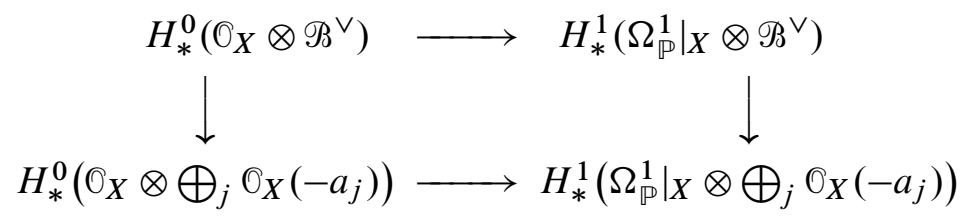

Then any minimal generator of the module $H_{*}^{0}\left(\mathscr{O}_{X} \otimes \mathscr{B}^{\vee}\right)$ maps to a nongenerator in $H_{*}^{0}\left(O_{X} \otimes \bigoplus_{j} O_{X}\left(-a_{j}\right)\right)$, and hence maps to zero in $H_{*}^{1}\left(\left.\Omega_{\mathbb{P}}^{1}\right|_{X} \otimes \bigoplus_{j} \mathrm{O}_{X}\left(-a_{j}\right)\right)=$ $\bigoplus_{j} k\left(-a_{j}\right)$. Thus the image of $H_{*}^{0}\left(\mathscr{O}_{X} \otimes \mathscr{B}^{\vee}\right)$ in $H_{*}^{1}\left(\left.\Omega_{\mathbb{P}}^{1}\right|_{X} \otimes \mathscr{B}^{\vee}\right)$ is nonzero and consists of $\mathscr{B}$-socle elements for $\left.\Omega_{\mathbb{P}}^{1}\right|_{X}$. So, for any ACM bundle $\mathscr{B}$ on $X$, without free summands, the Horrocks data bundle $\left.\Omega_{\mathbb{P}}^{1}\right|_{X}$ will have $\mathscr{B}$-socle elements.

For a general ACM variety $X$, one would expect infinitely many families of nonisomorphic and irreducible ACM bundles; hence this shows that even for a fixed Horrocks data bundle $\mathscr{F}_{\mathrm{min}}$, the number of bundles $\mathscr{E}$ with Horrocks datum $\left(\mathscr{F}_{\min }, \beta_{\mathscr{E}}\right)$ would get out of control, especially with the construction given below. In later sections, we will limit our attention to the quadric hypersurface and the Veronese surface, where there are only finitely many ACM bundles. In these sections, we will also be able to deal with arbitrary submodules of $\mathscr{B}$-socle elements, instead of the entire $\mathscr{B}$-socle module of the rather crude theorem below.

Theorem 1.15 (existence). Let $\mathscr{F}_{\min }$ be a minimal Horrocks data bundle on $X$, and let $\mathscr{B}_{1}, \mathscr{B}_{2}, \ldots, \mathscr{B}_{k}$ be a finite collection of irreducible, nonfree ACM bundles on $X$. Then there is a vector bundle $\mathscr{E}$ on $X$ with Horrocks datum $\left(\mathscr{F}_{\min }, \beta\right)$ and with ker $H_{*}^{1}\left(\beta \otimes 1_{\mathscr{B}_{i}^{\vee}}\right)=H_{*}^{1}\left(\mathscr{F}_{\min } \otimes \mathscr{B}_{i}^{\vee}\right)_{\text {soc }}$ for $1 \leq i \leq k$.

Proof. Each $H_{*}^{1}\left(\mathscr{F}_{\min } \otimes \mathscr{B}_{i}^{\vee}\right)_{\text {soc }}$ is an $A$-module, and we can pick a collection of minimal generators for the module. Let $K_{i}$ be the vector subspace spanned by this collection inside $H_{*}^{1}\left(\mathscr{F}_{\text {min }} \otimes \mathscr{B}_{i}^{\vee}\right)_{\text {soc }}$. Let $\mathscr{B}=\bigoplus\left(K_{i} \otimes_{k} \mathscr{B}_{i}\right)$. The data

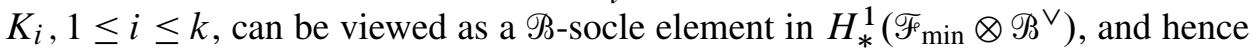
gives an extension (that defines a bundle $\mathscr{\mathscr { C }}$ )

$$
0 \longrightarrow \mathscr{F}_{\min } \stackrel{\beta}{\longrightarrow} \mathscr{E} \stackrel{\rho}{\longrightarrow} \mathscr{B} \longrightarrow 0 .
$$

Since the element is a socle element, the pullback of the sequence under any map $\mathcal{O}_{X}(b) \rightarrow \mathscr{B}$ will split. Hence $H_{*}^{0}(\rho)$ is surjective, giving $\left(\mathscr{F}_{\min }, \beta\right)$ the Horrocks datum for $\mathscr{E}$.

By construction, the subspace $K_{i} \cdot I_{\mathscr{B}_{i}}$ in $H_{*}^{0}\left(\mathscr{B} \otimes \mathscr{B}_{i}^{\vee}\right)$ maps isomorphically to $K_{i} \subseteq H_{*}^{1}\left(\mathscr{F}_{\min } \otimes \mathscr{P}_{i}^{\vee}\right)_{\text {soc }}$. Hence the image of the map of $A$-modules $H_{*}^{0}\left(\mathscr{B}_{B} \otimes \mathscr{B}_{i}^{\vee}\right) \rightarrow$ $H_{*}^{1}\left(\mathscr{F}_{\min } \otimes \mathscr{B}_{i}^{\vee}\right)_{\text {soc }}$ is onto. 
Remark 1.16. (1) The same construction can be done for an arbitrary $A$-submodule $V_{i}$ of $H_{*}^{1}\left(\mathscr{F}_{\min } \otimes \mathscr{B}_{i}^{\vee}\right)_{\text {soc }}$. We would choose $K_{i}$ to be the subspace spanned by a set of minimal generators for $V_{i}$. In the last step of the above proof, we find that image of the map of $A$-modules $H_{*}^{0}\left(\mathscr{B}_{B} \otimes \mathscr{B}_{i}^{\vee}\right) \rightarrow H_{*}^{1}\left(\mathscr{F}_{\min } \otimes \mathscr{B}_{i}^{\vee}\right)_{\text {soc }}$ contains $V_{i}$, and could possibly be larger. Hence the Horrocks invariants of $\mathscr{E}$, ker $H_{*}^{1}\left(\beta \otimes 1_{\mathscr{B}_{i}}\right)$, may not be precisely recognizable in this case.

(2) In the above theorem, for the $\mathscr{E}$ so constructed, it is possible to identify $A_{\mathscr{E}}$ in the case when $X$ is arithmetically Gorenstein, or when the dual of each of the ACM bundles $\mathscr{T}_{i}, 1 \leq i \leq k$, is also ACM: since the $\gamma$-sequence of $\mathscr{E}$ is the pushforward of the $\Psi$-sequence for $\mathscr{F}_{\text {min }}$, we get the exact sequence $0 \rightarrow \mathscr{P}^{0} \rightarrow \mathscr{A}_{\mathscr{E}} \rightarrow \mathscr{B} \rightarrow 0$, which is forced to split by the extra hypotheses. Once the ACM bundles in $\mathscr{A}_{\mathscr{E}}$ are identified, it is possible to compare $\mathscr{E}$ with other bundles via the uniqueness theorems (Theorems 1.10, 1.11).

(3) However, in the non-arithmetically Gorenstein case, a clear description of $A_{\mathscr{E}}$ may not be apparent at the end of the construction of the theorem. We will give an example later (Example 3.3) where an identification of $\mathscr{A}_{\mathscr{E}}$ requires more work.

It is easy to obtain a splitting criterion for a vector bundle $\mathscr{E}$ on $X$ to be free, which gives for example the criterion for quadrics in [Ancona and Ottaviani 1991] that was cited in the introduction. Once again, in the theorem below, note that the condition invoking any ACM bundle is not very useful when there are too many ACM bundles on $X$. It is more interesting (see the proof below) in the case where the choices for $\mathscr{B}$ are limited, for example, if one could limit the possible ACM bundles that might appear as a summand in the diagram of $\mathscr{E}$.

Theorem 1.17 (a splitting criterion). Let $\mathscr{E}$ be a vector bundle of rank $\leq r$ on $X$, a smooth ACM variety of dimension $n$, such that $H_{*}^{i}\left(\mathscr{E}^{\vee}\right)=0$ for $1 \leq i \leq$ $\min \{r-1, n-1\}$ and also $H_{*}^{1}\left(\mathscr{E}^{\vee} \otimes \mathscr{B}\right)=0$ for any $A C M$ bundle $\mathscr{B}$ on $X$. Then $\mathscr{E}$ is free.

Proof. Now the $\eta$-sequence (Theorem 1.7) of $\mathscr{E}, 0 \rightarrow \mathscr{K} \rightarrow \mathscr{F} \rightarrow \mathscr{E} \rightarrow 0$, gives an element in $H^{1}\left(\mathscr{E}^{\vee} \otimes \mathscr{K}\right)$ which is zero by hypothesis. Hence $\mathscr{K}$ and $\mathscr{E}$ are summands of $\mathscr{F}$. Since $\mathscr{F}$ is a Horrocks data bundle, it can have no nonfree ACM summand, so $\mathscr{K}$ must be free. Thus $\mathscr{E}$ itself is a Horrocks data bundle.

If $r \geq n, \mathscr{E} \vee$ is ACM. But the dual of a Horrocks data bundle has finite resolution, so $\mathscr{E}^{\vee}$ must be free.

If $r<n$, consider the sequence (3) with $\mathscr{E}$ replaced by $\mathscr{E} \vee$. From the vanishing of cohomologies of $\mathscr{E}^{\mathrm{V}}$, when we look at the complex of global sections of the sequence, we conclude that the module $E^{\vee}$ is an $(r+1)$-th syzygy, and $E^{\vee}$ has finite projective dimension since $\mathscr{E}$ is a Horrocks data bundle. By the Evans-Griffith syzygy theorem [1981], $\mathscr{E}^{\vee}$ is free. 
Remark 1.18. If $X$ is a smooth quadric hypersurface, the above splitting criterion is also equivalent to Corollary 4.3 of [Ballico and Malaspina 2009]. Splitting criteria have been established on other varieties. For a Grassmannian of lines $G(1, n)$, which supports infinitely many irreducible ACM bundles when $n \geq 4$, it is possible to prove a splitting criterion (see Theorem 2.6 of [Arrondo and Malaspina 2010]) with a finite number of cohomological vanishing conditions involving only the ACM bundles $S^{i} Q$, where $i=1, \ldots, n-2$ and $Q$ is the tautological rank-two bundle. Similarly, on multiprojective spaces $\mathbb{P}^{n_{1}} \times \cdots \times \mathbb{P}^{n_{s}}$, there is a splitting criterion (see Theorem 3.9 of [Ballico and Malaspina 2011]) with a finite number of cohomological vanishing conditions involving only the ACM bundles $\mathcal{O}\left(k_{1}, \ldots, k_{s}\right)$, where $-n_{j} \leq k_{j} \leq 0$. These results are much stronger than Theorem 1.17. Due to the generality of our setting, we are unable to prove a splitting criterion with conditions involving only a finite number of ACM bundles.

However, when there is additional analysis of the ACM bundles, more can be said. For example, Arrondo and Graña [1999] identified a list of six specific ACM bundles on $G(1,4)$, and showed that any other ACM bundle $\mathscr{B}$ is a summand of a bundle that appears in the middle of a short exact sequence of bundles, where the bundles on either side are built from direct sums of twists of these six bundles. Hence in our Theorem 1.17, applied to $G(1,4)$, it suffices to consider only these six specific bundles for $\mathscr{B}$. It is now straightforward to check that Ottaviani's splitting criterion on $G(1,4)$ (which is just one case of [Ottaviani 1987, Théorème 1]) follows from Theorem 1.17. (He assumed that $H_{*}^{i}(\mathscr{E} \vee)=0$ for $1 \leq i \leq 5$ and his other hypotheses imply that $H_{*}^{1}(\mathscr{E} \vee \otimes \mathscr{B})=0$ for these six ACM bundles.)

\section{Quadric hypersurfaces}

Let $2_{n} \subset \mathbb{P}^{n+1}$ be a smooth quadric hypersurface. We will work over a field of characteristic not two. The quadratic form defining $2_{n}$ descends to a quadratic form on the tangent bundle of $2_{n}$. Hence one can define spinor bundles on $2_{n}$ [Karrer 1973]. Set $l:=\lfloor(n+1) / 2\rfloor$. If $n$ is even, then $2_{n}$ has two distinct spinor bundles $\Sigma_{1}$ and $\Sigma_{2}$ of rank $2^{l-1}$. If $n$ is odd, then $2_{n}$ has a unique spinor bundle, which we denote $\Sigma_{1}$, of rank $2^{l-1}$. Algebraic properties of these bundles were studied by Ottaviani [1988], who obtained them using the geometry of the variety of all maximal linear subspaces of $2_{n}$ to construct morphisms from $2_{n}$ to $G\left(2^{l-1}, 2^{l}\right)$. He shows that these spinor bundles on $2_{n}$ are ACM bundles. Kapranov [1988] showed how these bundles were crucial in describing the derived category of sheaves on the quadric. Meanwhile, Knörrer [1987], classifying maximal Cohen-Macaulay modules over isolated quadratic hypersurface singularities, described these bundles as the fundamental ACM bundles on $2_{n}$ (see [Buchweitz et al. 1987] for the 
interpretation of Knörrer's results in terms of bundles). Knörrer's classification of ACM bundles on $2_{n}$ was proved also in [Ancona and Ottaviani 1991].

We use a unified notation $\Sigma_{i}$ for spinor bundles on $2_{n}$, where for even $n, i$ can take on the values 1,2, while if $n$ is odd, $i$ can be only 1 . We follow the notation of [Kapranov 1988], whose spinor bundles differ from those in [Ottaviani 1988] by a twist of 1 . Hence $\Sigma_{i}$ is generated by its global sections and $\Sigma_{i}(-1)$ has no sections.

We will call a bundle of the form $\Sigma_{i}(a)$ a twisted spinor bundle on $2_{n}$. The fundamental theorem of [Knörrer 1987] is:

Theorem 2.1. Any ACM bundle on $2_{n}$ is a direct sum of line bundles and twisted spinor bundles.

The spinor bundles on $2_{n}$ satisfy some dualities [Ottaviani 1988]: when $n$ is odd or $n \equiv 0(\bmod 4), \Sigma_{i}^{\vee} \cong \Sigma_{i}(-1)$, while if $n \equiv 2(\bmod 4), \Sigma_{i}^{\vee} \cong \Sigma_{j}(-1)$, where $j \neq i$.

In addition, the spinor bundles on $2_{n}$ satisfy canonical sequences. To further unify the notation, when $n$ is odd or when $n \equiv 2(\bmod 4)$, define $i \mapsto \bar{i}$ to be the identity on indices, and when $n \equiv 0(\bmod 4)$, define $i \mapsto \bar{i}$ to be the transposition of the indices 1 and 2. With this notation, we have the canonical sequences

$$
0 \longrightarrow \Sigma_{\bar{i}}^{\vee} \stackrel{u_{i}}{\longrightarrow} 0^{\oplus 2^{l}} \stackrel{v_{i}}{\longrightarrow} \Sigma_{i} \longrightarrow 0
$$

(see [Ottaviani 1988, Theorem 2.8]).

Ottaviani [1988, Lemma 2.7] proved that, for any spinor bundle $\Sigma_{i}, \operatorname{End}\left(\Sigma_{i}\right)=$ $H^{0}\left(\Sigma_{i} \otimes \Sigma_{i}^{\vee}\right)=k$ and $\operatorname{Hom}\left(\Sigma_{i}, \Sigma_{j}\right)=0$ for $i \neq j$. Using this, and tensoring the sequence above with $\Sigma_{i}^{\vee}$, we get $H^{1}\left(\Sigma_{\bar{i}}^{\vee} \otimes \Sigma_{i}^{\vee}\right)=k$, where $\operatorname{Id}_{\Sigma_{i}}$ maps to a generator of $H^{1}\left(\Sigma_{\bar{i}}^{\vee} \otimes \Sigma_{i}^{\vee}\right)$. For completeness, the following lemma is also easy to prove:

Lemma 2.2.

$$
\begin{aligned}
& H_{*}^{1}\left(\Sigma_{\bar{i}}^{\vee} \otimes \Sigma_{i}^{\vee}\right)=k, \\
& H_{*}^{1}\left(\Sigma_{j}^{\vee} \otimes \Sigma_{i}^{\vee}\right)=0 \quad \text { if } j \neq \bar{i} .
\end{aligned}
$$

Recall the definition of socle elements.

Definition 2.3. Let $\mathscr{F}$ be a sheaf on $2_{n}$. The sequence dual to (4) tensored by $\mathscr{F}_{F}$ gives

$$
0 \longrightarrow \mathscr{F} \otimes \Sigma_{i}^{\vee} \longrightarrow \mathscr{F} \otimes \mathcal{O}^{\oplus 2^{l}} \longrightarrow \mathscr{F} \otimes \Sigma_{\bar{i}} \longrightarrow 0
$$

and a natural map $H_{*}^{1}\left(\mathscr{F} \otimes \Sigma_{i}^{\vee}\right) \rightarrow H_{*}^{1}\left(\mathscr{F}_{F} \otimes \mathcal{O}^{\oplus 2^{l}}\right)$.

An element in $H^{1}\left(\mathscr{F}(d) \otimes \Sigma_{i}^{\vee}\right)$ will be called a $\Sigma_{i}$-socle element for $\mathscr{F}_{F}$ in degree $d$ if it is annihilated by the map $H^{1}\left(\mathscr{F}(d) \otimes \Sigma_{i}^{\vee}\right) \rightarrow H_{*}^{1}\left(\mathscr{F}_{F} \otimes \mathcal{O}^{\oplus 2^{l}}\right)$. 
The terminology "socle" comes from the case of a quadric surface studied in [Malaspina and Rao 2014], where socle elements were annihilated by multiplication by the forms lifted from one of the $\mathbb{P}^{1}$ factors of $2_{2}$. We have extended this terminology to all ACM bundles in Section 1.

Lemma 2.4. Let $\mathscr{F}$ be a sheaf on $2_{n}$. Let $V$ be a finite-dimensional graded subspace consisting of $\Sigma_{i}$-socle elements in $H_{*}^{1}\left(\mathscr{F} \otimes \Sigma_{i}^{\vee}\right)$. Then there is a homomorphism

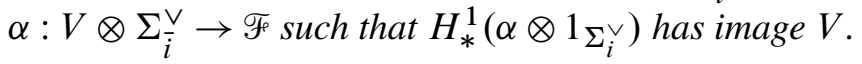

Proof. Consider the dual canonical sequence (4) tensored by $\mathscr{F}$

$$
0 \longrightarrow \mathscr{F}_{F} \otimes \Sigma_{i}^{\vee} \longrightarrow \mathscr{F} \otimes \mathrm{O}^{\oplus 2^{l}} \longrightarrow \mathscr{F}_{\mathcal{F}} \otimes \Sigma_{\bar{i}} \longrightarrow 0 .
$$

We get

$$
H^{0}\left(\mathscr{F} \otimes \Sigma_{\bar{i}}\right) \rightarrow H^{1}\left(\mathscr{F} \otimes \Sigma_{i}^{\vee}\right) \rightarrow H^{1}\left(\mathscr{F} \otimes \mathcal{O}^{\oplus 2^{l}}\right) .
$$

There is a graded subspace $V^{\prime}$ of $H_{*}^{0}\left(\mathscr{F}_{F} \otimes \Sigma_{\bar{i}}\right)$ which is mapped isomorphically to $V \subset H_{*}^{1}\left(\mathscr{F}_{F} \otimes \Sigma_{i}^{\vee}\right)$. This induces a map $\alpha: V^{\prime} \otimes_{k} \Sigma_{\bar{i}}^{\vee} \rightarrow \mathscr{F}_{F}$. Thus we can construct the commuting diagram

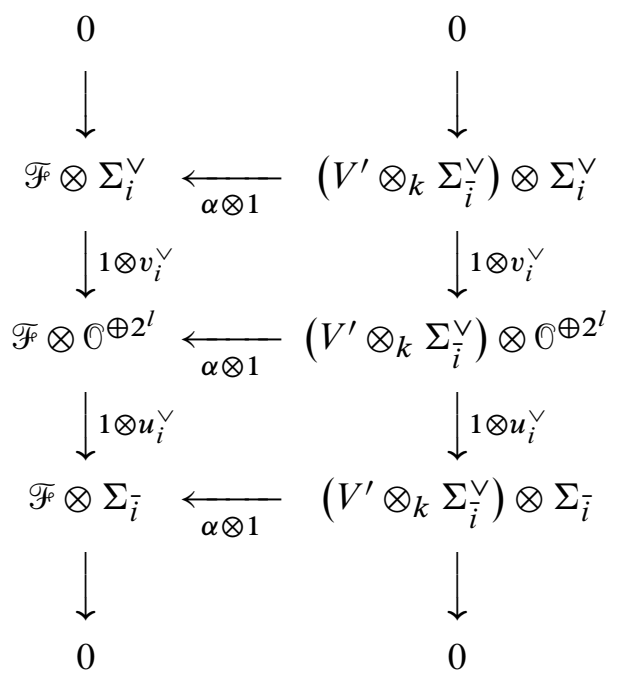

Then $H_{*}^{1}(\alpha \otimes 1): H_{*}^{1}\left(\left(V^{\prime} \otimes_{k} \Sigma_{\bar{i}}^{\vee}\right) \otimes \Sigma_{i}^{\vee}\right) \rightarrow H_{*}^{1}\left(\mathscr{F} \otimes \Sigma_{i}^{\vee}\right)$ gives $V^{\prime} \cong V$.

Corollary 2.5. Let F $\mathscr{F}_{\text {be }}$ a vector bundle on $2_{n}$. Then any graded vector subspace $V$ of $\Sigma_{i}$-socle elements in $H_{*}^{1}\left(\mathscr{F} \otimes \Sigma_{i}^{\vee}\right)_{\mathrm{soc}}$ is an A-submodule of $H_{*}^{1}\left(\mathscr{F} \otimes \Sigma_{i}^{\vee}\right)_{\mathrm{soc}}$. Proof. In the proof above $H_{*}^{1}\left(\alpha \otimes 1_{\Sigma_{i}^{\vee}}\right)$ is an $A$-module homomorphism, and by Lemma 2.2 the $A$-module $H_{*}^{1}\left(\left(V^{\prime} \otimes_{k} \Sigma_{\bar{i}}^{\vee}\right) \otimes \Sigma_{i}^{\vee}\right)$ has the trivial $A$-module structure, where multiplication by graded elements in $A$ of positive degree is zero.

For any vector bundle $\mathscr{E}$ on $\mathscr{2}_{n}$, we will define invariants as follows: 
Definition 2.6 (Horrocks invariants of $\mathscr{E}$ ). Let $\mathscr{E}$ be a vector bundle on $2_{n}$. It has a minimal associated Horrocks datum $\left(\mathscr{F}_{\min }, \beta\right)$. Let

$$
V_{i}=\operatorname{ker} H^{1}\left(\beta \otimes \operatorname{Id}_{\Sigma_{i}^{\vee}}\right): H_{*}^{1}\left(\mathscr{F}_{\min } \otimes \Sigma_{i}^{\vee}\right) \rightarrow H_{*}^{1}\left(\mathscr{E} \otimes \Sigma_{i}^{\vee}\right) .
$$

Then $V_{i}$ is a graded subspace of $H_{*}^{1}\left(\mathscr{F}_{\min } \otimes \Sigma_{i}^{\vee}\right)_{\text {soc }}$. The collection $\left(\mathscr{F}_{\min }, V_{i}\right)$ will be called Horrocks invariants for $\mathscr{E}$. (As usual, when $n$ is even, this means ( $\left.\mathscr{F}_{\min }, V_{1}, V_{2}\right)$ and when $n$ is odd, it means $\left(\mathscr{F}_{\min }, V_{1}\right)$.)

Remark 2.7. (1) $\mathscr{E}$ is ACM if and only if $\mathscr{F}_{\text {min }}$ is the zero bundle. $V_{i}=0$ as well.

(2) In general, $V_{i}=0$ for all $i$ if and only if $\mathscr{E}$ is a direct sum of a Horrocks data bundle and an ACM bundle.

(3) If $\mathscr{B}$ is an ACM bundle, then $\mathscr{E}$ and $\mathscr{E} \oplus \mathscr{B}$ will have the same Horrocks invariants.

(4) If ( $\left.\mathscr{F}_{\min }, \beta, V_{i}\right)$ is a collection of Horrocks invariants for $\mathscr{E}$ and $\phi$ is an automorphism of $\mathscr{F}_{\text {min }}$, then $\phi$ can be used to change $\beta: \mathscr{F}_{\text {min }} \rightarrow \mathscr{E}$ and hence also $V_{i}$ to get a new collection of Horrocks invariants for $\mathscr{E}$.

(5) The definition could have used an arbitrary Horrocks data bundle $\mathscr{F}$ for $\mathscr{E}$ instead of the minimal one $\mathscr{F}_{\text {min }}$, since $H_{*}^{1}\left(\Sigma_{i}^{\vee}\right)=0$ and hence the description of $V_{i}$ would not change.

A stronger existence theorem for quadrics can now be stated than was proved in Theorem 1.15. Below we have a statement that deals with arbitrary subspaces of socle elements:

Theorem 2.8 (existence). Let $\mathscr{F}_{\min }$ be a minimal Horrocks data bundle on $2_{n}$ and let $V_{i}$ be a graded vector subspace of $H_{*}^{1}\left(\mathscr{F}_{\min } \otimes \Sigma_{i}^{\vee}\right)_{\mathrm{soc}}$. Then there exists a vector bundle $\mathscr{E}$ with the Horrocks invariants $\left(\mathscr{F}_{\min }, V_{1}, V_{2}\right)$ (when $n$ is even) and invariants $\left(\mathscr{F}_{\min }, V_{1}\right)$ (when $n$ is odd).

Proof. We follow the approach in Theorem 1.15. For notational convenience, assume $n$ is even, so $i=1,2$. Let $\mathscr{B}=\left(V_{1} \otimes_{k} \Sigma_{1}\right) \oplus\left(V_{2} \otimes_{k} \Sigma_{2}\right)$. As in the earlier proof, we obtain a short exact sequence (defining $\mathscr{E})$ :

$$
0 \longrightarrow \mathscr{F}_{\text {min }} \stackrel{\beta}{\longrightarrow} \mathscr{E} \stackrel{\rho}{\longrightarrow}\left(V_{1} \otimes_{k} \Sigma_{1}\right) \oplus\left(V_{2} \otimes_{k} \Sigma_{2}\right) \longrightarrow 0,
$$

where $\left(\mathscr{F}_{\min }, \beta\right)$ is a Horrocks datum for the bundle $\mathscr{E}$ so obtained. Our goal is now to show that the image of $H_{*}^{0}\left(\mathscr{B} \otimes \Sigma_{i}^{\vee}\right) \rightarrow H_{*}^{1}\left(\mathscr{F}_{\min } \otimes \Sigma_{i}^{\vee}\right)$ is $V_{i}$, whereas in the earlier proof we showed that it contained $V_{i}$. Let $\Sigma_{j}(a)$ be any summand in $\mathscr{B}$, and pick a nonzero section $s \in H^{0}\left(\Sigma_{j}(a) \otimes \Sigma_{i}^{\vee}(b)\right)$, or a map $s: \Sigma_{i}(-b) \rightarrow \Sigma_{j}(a)$. Then $a+b \geq 0$. The section $s \in H^{0}\left(\mathscr{B} \otimes \Sigma_{i}^{\vee}(b)\right)$ maps to zero in $H_{*}^{1}\left(\mathscr{F}_{\min } \otimes \Sigma_{i}^{\vee}\right)$ if and only if the pullback of the short exact sequence by the map $s: \Sigma_{i}(-b) \rightarrow \mathscr{B}$ is a split sequence. If $a+b>0$, by Lemma 2.2 the map $s: \Sigma_{i}(-b) \rightarrow \Sigma_{j}(a)$ 
factors through $\mathrm{O}^{\oplus 2^{l}}(a)$. The pullback of the short exact sequence by the map $0^{\oplus 2^{l}}(a) \rightarrow \Sigma_{j}(a) \subseteq \mathscr{B}$ splits since the extension is defined by socle elements. Hence so does the pullback by the map $\Sigma_{i}(-b) \rightarrow \Sigma_{j}(a) \subseteq \mathscr{B}$.

It follows that the only nonzero contribution from this summand $\Sigma_{j}(a)$ to the image of $H^{0}\left(\mathscr{B} \otimes \Sigma_{i}^{\vee}(b)\right)$ occurs when $a+b=0$. If $i \neq j, \operatorname{Hom}\left(\Sigma_{i}, \Sigma_{j}\right)=0$ and so no section $s$ can be found. If $i=j, \operatorname{End}\left(\Sigma_{i}\right)=k$ and it follows that the image of $s$ lies in $V_{i}$. Thus the image of $H_{*}^{0}\left(\mathscr{B} \otimes \Sigma_{i}^{\vee}\right)$ is exactly $V_{i}$.

As pointed out after Theorem 1.15, if $\mathscr{F}_{\text {min }}$ has a $\Psi$-sequence $0 \rightarrow \mathscr{F}_{\text {min }} \rightarrow \mathscr{P}^{0} \rightarrow$ $\mathscr{G}_{\min } \rightarrow 0$, then the $\mathscr{E}$ constructed in the above theorem has $\gamma$-sequence given as

$$
0 \longrightarrow \mathscr{E} \longrightarrow \bigoplus_{i}\left(V_{i} \otimes_{k} \Sigma_{i}\right) \oplus \mathscr{P}^{0} \longrightarrow \mathscr{G}_{\min } \longrightarrow 0 .
$$

It is also easy to see that since $\mathscr{F}_{\min }$ has no summands of type $\Sigma_{i}$, neither does $\mathscr{E}$. Conversely, suppose $\mathscr{E}$ is a vector bundle on $\mathscr{2}_{n}$ with Horrocks invariants $\left(\mathscr{F}_{\min }, V_{i}\right)$ and with no summands of type $\Sigma_{i}$. It will follow from the next theorems that $\mathscr{E}$ has a $\gamma$-sequence with $\mathscr{A}_{\mathscr{E}}=\bigoplus_{i}\left(V_{i} \otimes_{k} \Sigma_{i}\right) \oplus \mathscr{P}^{\prime}$, where $\mathscr{P}^{\prime}$ is free.

The following two uniqueness results follow easily from the general theorems of Section 1.

Theorem 2.9 (uniqueness). Given two bundles $\mathscr{E}, \mathscr{E}$ ' on $2_{n}$ without ACM summands and with Horrocks invariants $\left(\mathscr{F}_{\min }, V_{i}\right),\left(\mathscr{F}_{\min }^{\prime}, V_{i}^{\prime}\right)$, suppose that there exists $\phi: \mathscr{F}_{\text {min }} \stackrel{\sim}{\longrightarrow} \mathscr{F}_{\text {min }}^{\prime}$ such that the induced isomorphisms $H_{*}^{1}\left(\mathscr{F}_{\min } \otimes \Sigma_{i}^{\vee}\right) \cong$ $H_{*}^{1}\left(\mathscr{F}_{\min }^{\prime} \otimes \Sigma_{i}^{\vee}\right)$ carry $V_{i}$ to $V_{i}^{\prime}$ for each $i$. Then $\mathscr{E}$ and $\mathscr{E}^{\prime}$ are isomorphic.

Proof. We may assume that $\mathscr{E}$ and $\mathscr{C}^{\prime}$ have the same minimal Horrocks data bundle $\mathscr{F}_{\text {min }}$. If $\mathscr{F}_{\text {min }}$ is zero, $\mathscr{E}, \mathscr{E}^{\prime}$ are ACM and the theorem does not apply. So we will assume that $\mathscr{F}_{\min }$ is a nonfree minimal Horrocks data bundle. If $V_{i}$ is 0 for $i=1,2$, then $\mathscr{E}$ is stably equivalent to $\mathscr{F}_{\text {min }}$, and, being without ACM summands, it must be isomorphic to $\mathscr{F}_{\min }$. Since $V_{i}^{\prime}$ will also be zero, the same is true for $\mathscr{E}^{\prime}$ and we conclude that $\mathscr{E} \cong \mathscr{E}^{\prime}$. So assume $V_{i}$ is nonzero for some $i$. If there is an automorphism $\phi$ of $\mathscr{F}_{\text {min }}$ which carries $V_{i}$ to $V_{i}^{\prime}$, in the diagram of Theorem 1.8 for $\mathscr{E}^{\prime}$, we may replace $\beta^{\prime}: \mathscr{F}_{\text {min }} \rightarrow \mathscr{E}^{\prime}$ by $\beta^{\prime} \circ \phi^{-1}$ and so on, and assume that $\beta$ and $\beta^{\prime}$ give the same kernel $V_{i}$ in $H_{*}^{1}\left(F_{\min } \otimes \Sigma_{i}^{\vee}\right)$.

We can now apply Theorem 1.10 to conclude the result.

Theorem 2.10. Let $\mathscr{E}, \mathscr{E}^{\prime}$ be vector bundles on $2_{n}$ with no ACM summands. Suppose $\sigma: \mathscr{E} \rightarrow \mathscr{E}^{\prime}$ is a homomorphism such that $\sigma$ induces $H_{*}^{j}(\mathscr{E}) \cong H_{*}^{j}\left(\mathscr{C}^{\prime}\right)$ for $1 \leq j \leq$ $n-1$ and also isomorphisms $H_{*}^{1}\left(\mathscr{E} \otimes \Sigma_{i}^{\vee}\right) \cong H_{*}^{1}\left(\mathscr{E}^{\prime} \otimes \Sigma_{i}^{\vee}\right)$ for all $i$. Then $\sigma$ is an isomorphism.

Proof. This is just Theorem 1.11 with the additional condition that $\mathscr{E}$ has no ACM summands. 


\section{The Veronese surface}

The Veronese surface $\mathscr{V} \subset \mathbb{P}^{5}$ is an arithmetically Cohen-Macaulay embedding which is not arithmetically Gorenstein. The study of vector bundles on $\mathscr{V}$ is trivial if we view $\mathscr{V}$ as $\mathbb{P}^{2}$. Below we discuss how the techniques of Section 1 apply to the embedded variety $\mathscr{V}$. With its polarization from the embedding, $\mathscr{V}$ has two irreducible, nonfree ACM bundles (up to twists). Hence, as in the case of quadric hypersurfaces of even dimension, we can define Horrocks invariants $\left(\mathscr{F}_{\min }, V, W\right)$ for any vector bundle $\mathscr{E}$ on $\mathscr{V}$. But unlike in the case of the quadric, where $V, W$ were independent of each other, here there is a dependency between them.

In the following discussion, we will write $\mathrm{O}_{\mathscr{V}}(1)$ for $\left.\mathrm{O}_{\mathbb{P}^{5}}(1)\right|_{\mathscr{V}}$ and $\mathrm{O}_{\mathscr{V}}(n)$ for $\operatorname{Or}_{\mathscr{V}}(1)^{\otimes n}$. We will write $\mathscr{L}$ for $\mathcal{O}_{\mathbb{p}^{2}}(1)$ and $U$ for $\Omega_{\mathscr{V}}^{1} \otimes \mathscr{L}$. Then the only irreducible ACM bundles on $\mathscr{V}$ (with respect to the polarization $\mathcal{O}_{\mathscr{V}}(1)$ ) are $\mathcal{O}_{\mathscr{V}}(n), \mathscr{L}(n)$ and $\mathcal{U}(n)$. In the diagram of a bundle $\mathscr{E}$ on $\mathscr{V}$ in Theorem 1.8 , the terms $\mathscr{A}_{\mathscr{E}}$ and $\mathscr{K}_{\mathscr{E}}$ are built out of these three types of irreducible ACM bundles. The vector bundle $\mathscr{G}$ is a free bundle and the $\Psi$-sequence is the sheafification of a free presentation of the $A$-module $H_{*}^{1}(\mathscr{E})$. The connection between $\mathscr{A}_{\mathscr{E}}$ and $\mathcal{K}_{\mathscr{E}}$, given by the $\Delta$-sequence in the diagram of $\mathscr{E}$, is controlled by the canonical sequences

$$
0 \longrightarrow u \stackrel{u}{\longrightarrow} 3 \mathrm{O}_{\mathrm{r}} \stackrel{v}{\longrightarrow} \mathscr{L} \longrightarrow 0
$$

and

$$
0 \longrightarrow 30(-1) \oplus \mathrm{O} \text { V }(-1) \longrightarrow 90 \%(-1) \longrightarrow u \longrightarrow 0,
$$

where the second can be simplified noncanonically to

$$
0 \longrightarrow 3 \cup(-1) \stackrel{u^{\prime}}{\longrightarrow} 8 \mathrm{O} \mathrm{v}(-1) \stackrel{v^{\prime}}{\longrightarrow} u \longrightarrow 0 .
$$

In addition, there is the canonical sequence

$$
0 \longrightarrow \operatorname{Or}(-1) \longrightarrow 3 \mathscr{L}(-1) \longrightarrow \vartheta \longrightarrow 0
$$

The two uniqueness theorems of Section 1 apply in this setting, where given a bundle $\mathscr{E}$ on $\mathscr{V}$ we can construct Horrocks invariants for $\mathscr{E}$ as $(\mathscr{F} \min , V, W)$, where $\left(\mathscr{F}_{\text {min }}, \beta\right)$ is a Horrocks datum for $\mathscr{E}, V=\operatorname{ker}\left(H_{*}^{1}\left(\mathscr{F}_{\min } \otimes \mathscr{L}^{\vee}\right) \rightarrow H_{*}^{1}\left(\mathscr{E} \otimes \mathscr{L}^{\vee}\right)\right)$ and $W=\operatorname{ker}\left(H_{*}^{1}\left(\mathscr{F}_{\min } \otimes \mathcal{U}^{\vee}\right) \rightarrow H_{*}^{1}\left(\mathscr{E} \otimes \mathcal{U}^{\vee}\right)\right)$. Thus to complete the classification of bundles on $\mathscr{V}$ by this method it remains to get a description of any constraints on $V \subseteq H_{*}^{1}\left(\mathscr{F}_{F} \otimes \mathscr{L}^{\vee}\right)$ and $W \subseteq H_{*}^{1}\left(\mathscr{F}_{\min } \otimes \mathcal{U}^{\vee}\right)$, and to finally show that given ( $\mathscr{F}_{\text {min }}, V, W$ ) with these constraints, there exists a bundle $\mathscr{E}$ with those invariants.

By Remark 1.13, $V$ is an $A$-submodule of $\mathscr{L}$-socle elements in $H_{*}^{1}\left(\mathscr{F}_{\min } \otimes \mathscr{L}^{\vee}\right)_{\text {soc }}$ and $W$ is an $A$-submodule of $U$-socle elements in $H_{*}^{1}\left(\mathscr{F}_{\text {min }} \otimes U^{\vee}\right)_{\text {soc }}$. By the next lemma, there is no distinction between the concepts of graded $A$-submodules and graded vector subspaces of socle elements: 
Lemma 3.1. For any vector bundle $\mathscr{F}_{\mathrm{F}}$ on $\mathcal{V}$, in the A-module structures of both $H_{*}^{1}\left(\mathscr{F}_{\mathrm{F}} \otimes \mathscr{L}^{\vee}\right)_{\mathrm{soc}}$ and $H_{*}^{1}\left(\mathscr{F} \otimes \mathcal{U}^{\vee}\right)_{\mathrm{soc}}$, multiplication by graded elements of positive degree in $A$ is zero.

Proof. Let $\eta \in H^{1}\left(\mathscr{F}(d) \otimes \mathscr{L}^{\vee}\right)_{\text {soc }}$, giving a short exact sequence $0 \rightarrow \mathscr{F}(d) \rightarrow \mathscr{A} \rightarrow$ $\mathscr{L} \rightarrow 0$. Consider multiplication by $x \in A$ of degree one, $\cdot x: \mathscr{L}(-1) \rightarrow \mathscr{L}$. The pullback by this map of the short exact sequence (7) is split since $H^{1}\left(\mathcal{U} \otimes \mathscr{L}^{\vee}(1)\right)=0$. So $\cdot x: \mathscr{L}(-1) \rightarrow \mathscr{L}$ factors through 30 q. By the definition of $\mathscr{L}$-socle elements, the pullback of $\eta$ by 30r $\rightarrow \mathscr{L}$ splits, hence also the pullback of $\eta$ by $\cdot x: \mathscr{L}(-1) \rightarrow \mathscr{L}$. Thus $x \cdot \eta=0$.

A similar proof works for an element $\eta \in H^{1}\left(\mathscr{F}(d) \otimes U^{\vee}\right)_{\text {soc. }}$ One notices that the pullback by $\cdot x: U(-1) \rightarrow U$ of the short exact sequence (9) is split because $H_{*}^{1}\left(u \otimes u^{\vee}\right)=3 k$ supported in $H^{1}\left(U \otimes U^{\vee}(-1)\right)$.

In the definition of $U$-socle elements for $\mathscr{F}$, the noncanonical inclusion $\mathcal{U}^{\vee} \hookrightarrow$ $80 \%$ (1) can be replaced by a canonical composite inclusion $\mathcal{U}^{\vee} \hookrightarrow 3 \mathscr{L}^{\vee}(1) \hookrightarrow$ 90\% (1). For any bundle $\mathscr{F}$, this gives a canonical map

$$
\phi_{\mathscr{F}}: H_{*}^{1}\left(\mathscr{F} \otimes U^{\vee}\right)_{\mathrm{soc}} \rightarrow 3 H_{*}^{1}\left(\mathscr{F}(1) \otimes \mathscr{L}^{\vee}\right)_{\mathrm{soc}} .
$$

When $\mathscr{E}$ is a vector bundle with Horrocks invariants $\left(\mathscr{F}_{\min }, V, W\right)$, it is immediate to see that $V$ and $W$ are related by $\phi_{\mathscr{F}_{\text {min }}}(W) \subseteq 3 V(1)$. This is a dependency between $V$ and $W$. In fact, this is the only requirement on the pair $(V, W)$ for proving an existence theorem on the Veronese surface:

Theorem 3.2. Let $\mathscr{F}_{\min }$ be a minimal Horrocks data bundle on $\mathscr{V}$, and let $V, W$ be graded vector subspaces of $H_{*}^{1}\left(\mathscr{F}_{\min } \otimes \mathscr{L}^{\vee}\right)_{\mathrm{soc}}, H_{*}^{1}\left(\mathscr{F}_{\min } \otimes \mathcal{U}^{\vee}\right)_{\mathrm{soc}}$ with the property that $\phi_{\mathscr{F}_{\min }}(W) \subseteq 3 V(1)$. Then there is a vector bundle $\mathscr{E}$ on $\mathscr{V}$ with Horrocks invariants $\left(\mathscr{F}_{\min }, V, W\right)$.

Proof. Construct $\mathscr{E}$ as an extension of $\mathscr{F}_{\text {min }}$ by $\mathscr{B}=\left(V \otimes_{k} \mathscr{L}\right) \oplus\left(W \otimes_{k} U\right)$ :

$$
0 \longrightarrow \mathscr{F}_{\min } \stackrel{\beta}{\longrightarrow} \mathscr{E} \longrightarrow \mathscr{B} \longrightarrow 0 .
$$

Since $V, W$ are subspaces of socle elements, $\mathscr{E}$ has $\left(\mathscr{F}_{\min }, \beta\right)$ as its Horrocks datum. We wish to understand the images of $H_{*}^{0}\left(\mathscr{B} \otimes \mathscr{L}^{\vee}\right) \rightarrow H_{*}^{1}\left(\mathscr{F}_{\min } \otimes \mathscr{L}^{\vee}\right)$ and $H_{*}^{0}\left(\mathscr{B} \otimes \mathcal{U}^{\vee}\right) \rightarrow H_{*}^{1}\left(\mathscr{F}_{\min } \otimes \mathcal{U}^{\vee}\right)$. $\operatorname{End}(\mathscr{L})=\operatorname{End}(\mathcal{U})=k$ and the image of $V \cdot I_{\mathscr{L}} \subseteq H^{0}\left(V \otimes \mathscr{L} \otimes \mathscr{L}^{\vee}\right)$ and $W \cdot I_{u} \subseteq H^{0}\left(W \otimes U \otimes U^{\vee}\right)$ give $V$ and $W$ in $H_{*}^{1}\left(\mathscr{F}_{\min } \otimes \mathscr{L}^{\vee}\right)_{\text {soc }}$ and $H_{*}^{1}\left(\mathscr{F}_{\text {min }} \otimes \mathcal{U}^{\vee}\right)_{\text {soc }}$. It remains to analyze any other contributions to the two images inside $H_{*}^{1}\left(\mathscr{F}_{\min } \otimes \mathscr{L}^{\vee}\right)_{\mathrm{soc}}$ and $H_{*}^{1}\left(\mathscr{F}_{\min } \otimes \mathcal{U}^{\vee}\right)_{\mathrm{soc}}$, and prove that the images are just $V$ and $W$ respectively.

Let $\mathscr{L}(b), \mathcal{U}(b)$ be any summands in $\left(V \otimes_{k} \mathscr{L}\right) \oplus\left(W \otimes_{k} \cup\right)$. Consider maps $\sigma_{1}: \mathscr{L}(a) \rightarrow \mathscr{L}(b), \sigma_{2}: \mathscr{L}(a) \rightarrow \mathcal{U}(b), \sigma_{3}: \mathcal{U}(a) \rightarrow \mathcal{U}(b), \sigma_{4}: U(a) \rightarrow \mathscr{L}(b)$. For $\sigma_{1}$, assume $a<b$ since we wish to omit endomorphisms of $\mathscr{L}$. Likewise for $\sigma_{3}$. In 
the sequence (7) tensored by $\mathscr{L}^{\vee}(b-a)$ we have $H^{1}\left(U \otimes \mathscr{L}^{\vee}(b-a)\right)=0$, and in the sequence (9) tensored by $u^{\vee}(b-a)$ we have $H^{1}\left(3 u(-1) \otimes u^{\vee}(b-a)\right)=0$. Hence $\sigma_{1}$ factors through $30_{\gamma}(b)$ and $\sigma_{3}$ factors through $80_{\sigma}(b-1)$. By the socle nature of the extension $(*)$, pullbacks of $(*)$ by $\sigma_{1}$ and $\sigma_{3}$ split; hence the element $\sigma_{1} \in H^{0}\left(\mathscr{L}(b) \otimes \mathscr{L}^{\vee}(-a)\right)$ maps to zero in $H_{*}^{1}\left(\mathscr{F}_{\min } \otimes \mathscr{L}^{\vee}\right)$, and likewise $\sigma_{3}$ maps to zero in $H_{*}^{1}\left(\mathscr{F}_{\min } \otimes U^{\vee}\right)$.

For $\sigma_{4}$ to be nonzero, we require that $a<b+1$. We know $H^{1}\left(u \otimes u^{\vee}(b-a)\right)=0$. Hence the same argument applies to show that $\sigma_{4}$ factors through 30 $\mathrm{r}(b)$, and we are done. The arguments for $\sigma_{3}, \sigma_{4}$ show that the image of $H_{*}^{0}\left(\mathscr{B} \otimes U^{\vee}\right) \rightarrow$ $H_{*}^{1}\left(\mathscr{F}_{\min } \otimes U^{\vee}\right)$ equals $W$.

For $\sigma_{2}$ to be nonzero we require that $a<b$, and we know that

$$
H^{1}\left(3 \cup(-1) \otimes \mathscr{L}^{\vee}(b-a)\right)=0
$$

except when $b-a=1$. Hence the only situation of difficulty is when we have $\sigma_{2}: \mathscr{L}(b-1) \rightarrow \mathcal{U}(b)$. Suppose the pullback of our short exact sequence $(*)$ by $\mathscr{L}(b-1) \stackrel{\sigma_{2}}{\longrightarrow} U(b) \hookrightarrow \mathscr{B}$ is nonsplit. The pullback of $(*)$ by $U(b) \hookrightarrow \mathscr{B}$ gives a nonzero element $w$ of degree $-b$ in $W \subseteq H_{*}^{1}\left(\mathscr{F}_{\min } \otimes \mathcal{U}^{\vee}\right)_{\text {soc. }}$. The nonsplit pullback by $\mathscr{L}(b-1) \rightarrow \mathscr{B}$ gives a nonzero element $v$ in $H^{1}\left(\mathscr{F}_{\min } \otimes \mathscr{L}^{\vee}(-b+1)\right)_{\text {soc }}$ which is the image of $w$ under $\sigma_{2}^{\vee}$. Since $\sigma_{2}^{\vee}$ is one component in $\mathcal{U}^{\vee}(-b) \hookrightarrow 3 \mathscr{L}^{\vee}(-b+1)$, the assumption that $\phi_{\mathscr{F}_{\text {min }}}(W) \subseteq 3 V(1)$ tells us that $v \in V$. Thus, the image of $H_{*}^{0}\left(\mathscr{B} \otimes \mathscr{L}^{\vee}\right) \rightarrow H_{*}^{1}\left(\mathscr{F}_{\min } \otimes \mathscr{L}^{\vee}\right)$ equals $V$.

We conclude with an example:

Example 3.3. The simplest non-ACM bundle on $\mathscr{V}$ is $\mathscr{E}=\Omega_{\mathscr{V}}^{1}=\mathscr{U} \otimes \mathscr{L}^{\vee}$, with $H_{*}^{1}(\mathscr{E})=k$ and $\gamma$-sequence $0 \rightarrow \mathscr{E} \rightarrow 3 \mathscr{L}^{\vee} \rightarrow \mathrm{O}_{\gamma} \rightarrow 0$, while its minimal Horrocks data bundle is $\mathscr{F}_{F}=\mathscr{F}_{\text {min }}=\left.\Omega_{\mathbb{p} 5}^{1}\right|_{\mathscr{q}}$, with $\Psi$ sequence $0 \rightarrow \mathscr{F} \rightarrow 6 \mathrm{O}_{\mathscr{V}}(-1) \rightarrow \mathrm{O}_{\mathscr{V}} \rightarrow 0$. The map $\beta: \mathscr{F} \rightarrow \mathscr{E}$ is the standard map $\left.\Omega_{\mathrm{p} 5}^{1}\right|_{\mathscr{V}} \rightarrow \Omega_{\mathscr{V}}^{1}$, which is a surjective map of vector bundles but not surjective on the module of global sections. The Horrocks invariants $(\mathscr{F}, V, W)$ of $\mathscr{E}$ are easy to work out and are described below.

$H_{*}^{1}\left(\mathscr{F} \otimes \mathscr{L}^{\vee}\right)=H^{1}\left(\mathscr{F}(1) \otimes \mathscr{L}^{\vee}\right)=3 k$, and $H_{*}^{1}\left(\mathscr{E} \otimes \mathscr{L}^{\vee}\right)=0$, hence $V=3 k=$ $H^{1}\left(\mathscr{F}(1) \otimes \mathscr{L}^{\vee}\right)$, where all elements in $H_{*}^{1}\left(\mathscr{F} \otimes \mathscr{L}^{\vee}\right)$ are $\mathscr{L}$-socle.

There is a commutative diagram that shows the only nonzero parts of $H_{*}^{1}\left(\mathscr{F} \otimes U^{\vee}\right)$ and $H_{*}^{1}\left(\mathscr{E} \otimes U^{\vee}\right)$

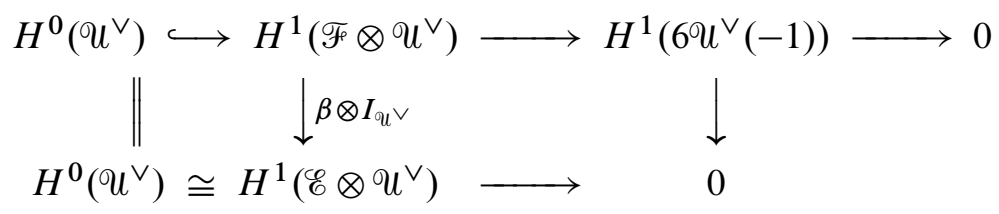

Hence $H_{*}^{1}\left(\mathscr{F} \otimes U^{\vee}\right)=H^{1}\left(\mathscr{F} \otimes U^{\vee}\right)$ is nine-dimensional, and the kernel $W$ of $H_{*}^{1}\left(\beta \otimes I U^{\vee}\right)$ is a six-dimensional subspace (of $U$-socle elements) that maps isomorphically to $H^{1}\left(6 u^{\vee}(-1)\right)$. 
When we apply the construction of the existence theorems (Theorems 1.15, 3.2) to the data $(\mathscr{F}, V, W)$, we obtain a vector bundle $\widetilde{\mathscr{E}}$ and a pushout diagram (refer to the discussion after Theorem 1.15)

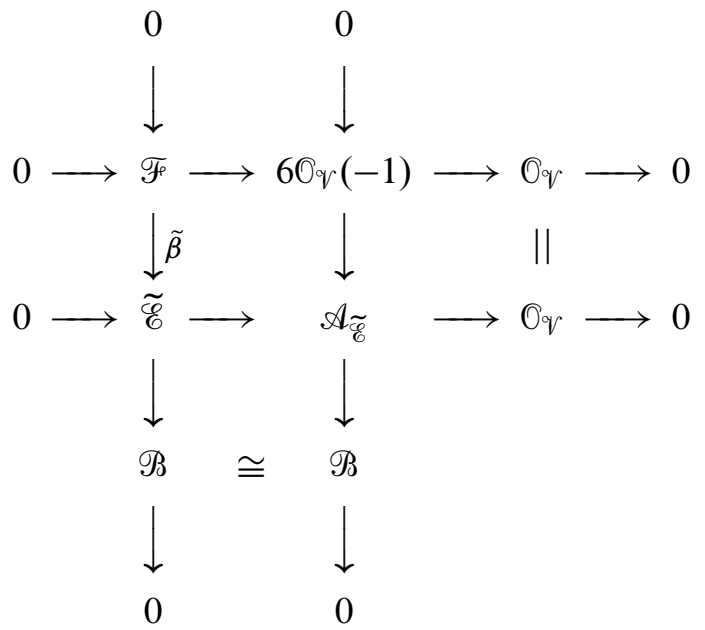

where $\mathscr{B}=\left(V \otimes_{k} \mathscr{L}\right) \oplus\left(W \otimes_{k} u\right)$.

According to the uniqueness theorems, $\mathscr{E}$ is a rank-two summand of the rank-20 bundle $\widetilde{\mathscr{E}}$, with the remaining summand of $\widetilde{\mathscr{E}}$ consisting of ACM bundles. In this example, even $\mathscr{A}_{\tilde{\tilde{g}}}$ is not obvious because the middle short exact sequence is not split. Indeed, the middle sequence is the pushout of the left sequence, hence it is split if and only if, under $\mathscr{F} \rightarrow 60_{\mathscr{V}}(-1)$, the image of the element $\tau \in H^{1}\left(\mathscr{F} \otimes \mathscr{B}^{\vee}\right)$ is zero in $H^{1}\left(60_{\mathscr{V}}(-1) \otimes \mathscr{B}^{\vee}\right)$. However, the components of $\tau$ in each of the U-summands of $\mathscr{B}$ generate the vector space $W \subset H^{1}\left(\mathscr{F} \otimes \mathcal{U}^{\vee}\right)$, and $W$ maps isomorphically to $H^{1}\left(6 U^{\vee}(-1)\right)$. Hence the image of $\tau$ is nonzero.

To understand $\widetilde{\mathscr{E}}$ and $A_{\tilde{E}}$, a little more work is needed. The fact that $W$ maps isomorphically to $H^{1}\left(6 u^{\vee}(-1)\right)$ tells us that the middle short exact sequence contains six copies of the canonical sequence (10). Hence $A_{\widetilde{\mathscr{C}}}=21 \mathscr{L}^{\vee}$. The map $A_{\tilde{\mathscr{C}}} \rightarrow \mathcal{O}_{\mathscr{V}}$ is now easy to understand and shows that $\widetilde{\mathscr{E}}=\mathscr{E} \oplus 18 \mathscr{L}^{\vee}$.

\section{References}

[Ancona and Ottaviani 1991] V. Ancona and G. Ottaviani, "Some applications of Beilinson's theorem to projective spaces and quadrics", Forum Math. 3:2 (1991), 157-176. MR 92e:14039 Zbl 0725.14009

[Arrondo and Graña 1999] E. Arrondo and B. Graña, "Vector bundles on $G(1,4)$ without intermediate cohomology", J. Algebra 214:1 (1999), 128-142. MR 2000e:14069 Zbl 0963.14027

[Arrondo and Malaspina 2010] E. Arrondo and F. Malaspina, "Cohomological characterization of vector bundles on Grassmannians of lines”, J. Algebra 323:4 (2010), 1098-1106. MR 2010m:14054 Zbl 1200.14081 
[Auslander and Bridger 1969] M. Auslander and M. Bridger, Stable module theory, Memoirs of the American Mathematical Society 94, American Mathematical Society, Providence, R.I., 1969. MR 42 \#4580 Zbl 0204.36402

[Ballico and Malaspina 2009] E. Ballico and F. Malaspina, "Qregularity and an extension of the EvansGriffiths criterion to vector bundles on quadrics", J. Pure Appl. Algebra 213:2 (2009), 194-202. MR 2009j:14055 Zbl 1153.14014

[Ballico and Malaspina 2011] E. Ballico and F. Malaspina, "Regularity and cohomological splitting conditions for vector bundles on multiprojective spaces”, J. Algebra 345 (2011), 137-149. MR 2842058 Zbl 1246.14056

[Beǔlinson 1978] A. A. Beب̌linson, "Coherent sheaves on $\mathbb{P}^{n}$ and problems in linear algebra", Funktsional. Anal. i Prilozhen. 12:3 (1978), 68-69. In Russian; translated in Funct. Anal. Appl. 12:3 (1978), 214-216. MR 80c:14010b

[Buchweitz 1986] R.-O. Buchweitz, "Maximal Cohen-Macaulay modules and Tate-Cohomology over Gorenstein rings", preprint, 1986, http://hdl.handle.net/1807/16682.

[Buchweitz et al. 1987] R.-O. Buchweitz, G.-M. Greuel, and F.-O. Schreyer, "Cohen-Macaulay modules on hypersurface singularities, II", Invent. Math. 88:1 (1987), 165-182. MR 88d:14005 Zbl 0617.14034

[Coandă 2010] I. Coandă, "The Horrocks correspondence for coherent sheaves on projective spaces", Homology, Homotopy Appl. 12:1 (2010), 327-353. MR 2012a:14036 Zbl 1197.14012

[Evans and Griffith 1981] E. G. Evans and P. Griffith, "The syzygy problem", Ann. of Math. (2) 114:2 (1981), 323-333. MR 83i:13006 Zbl 0497.13013

[Horrocks 1964] G. Horrocks, "Vector bundles on the punctured spectrum of a local ring", Proc. London Math. Soc. (3) 14 (1964), 689-713. MR 30 \#120 Zbl 0126.16801

[Kapranov 1988] M. M. Kapranov, "On the derived categories of coherent sheaves on some homogeneous spaces”, Invent. Math. 92:3 (1988), 479-508. MR 89g:18018 Zbl 0651.18008

[Karrer 1973] G. Karrer, "Darstellung von Cliffordbündeln", Ann. Acad. Sci. Fenn. Ser. A I 521 (1973), 34. MR 47 \#641 Zbl 0262.53025

[Knörrer 1987] H. Knörrer, “Cohen-Macaulay modules on hypersurface singularities, I", Invent. Math. 88:1 (1987), 153-164. MR 88d:14004 Zbl 0617.14033

[Malaspina and Rao 2014] F. Malaspina and A. P. Rao, "Horrocks correspondence on a quadric surface", Geom. Dedicata 169 (2014), 15-31. MR 3175233 Zbl 06309484

[Ottaviani 1987] G. Ottaviani, "Critères de scindage pour les fibrés vectoriels sur les Grassmanniennes et les quadriques”, C. R. Acad. Sci. Paris Sér. I Math. 305:6 (1987), 257-260. MR 88j:14024 Zbl 0629.14012

[Ottaviani 1988] G. Ottaviani, "Spinor bundles on quadrics", Trans. Amer. Math. Soc. 307:1 (1988), 301-316. MR 89h:14012 Zbl 0657.14006

[Walter 1996] C. H. Walter, "Pfaffian subschemes", J. Algebraic Geom. 5:4 (1996), 671-704. MR 99f:14064 Zbl 0864.14032

Communicated by David Eisenbud

Received 2014-10-06 Revised 2015-02-23 Accepted 2015-04-07

francesco.malaspina@polito.it Dipartimento di Scienze Matematiche, Politecnico di Torino, Corso Duca degli Abruzzi 24, I-10129 Torino, Italy

raoa@umsl.edu

Department of Mathematics, University of Missouri - St. Louis, Saint Louis, MO 63121, United States 


\section{Algebra \& Number Theory}

msp.org/ant

\section{EDITORS}

MANAGING EDITOR

Bjorn Poonen

Massachusetts Institute of Technology

Cambridge, USA

\author{
EDITORIAL BOARD CHAIR \\ David Eisenbud \\ University of California \\ Berkeley, USA
}

\section{BOARD OF EDITORS}

Georgia Benkart

Dave Benson

Richard E. Borcherds

John H. Coates

J-L. Colliot-Thélène

Brian D. Conrad

Hélène Esnault

Hubert Flenner

Edward Frenkel

Andrew Granville

Joseph Gubeladze

Roger Heath-Brown

Craig Huneke

Kiran S. Kedlaya

János Kollár

Yuri Manin

Barry Mazur

Philippe Michel
University of Wisconsin, Madison, USA

University of Aberdeen, Scotland

University of California, Berkeley, USA

University of Cambridge, UK

CNRS, Université Paris-Sud, France

Stanford University, USA

Freie Universität Berlin, Germany

Ruhr-Universität, Germany

University of California, Berkeley, USA

Université de Montréal, Canada

San Francisco State University, USA

Oxford University, UK

University of Virginia, USA

Univ. of California, San Diego, USA

Princeton University, USA

Northwestern University, USA

Harvard University, USA

École Polytechnique Fédérale de Lausanne
Susan Montgomery

Shigefumi Mori

Raman Parimala

Jonathan Pila

Anand Pillay

Victor Reiner

Peter Sarnak

Joseph H. Silverman

Michael Singer

Vasudevan Srinivas

J. Toby Stafford

Ravi Vakil

Michel van den Bergh

Marie-France Vignéras

Kei-Ichi Watanabe

Efim Zelmanov

Shou-Wu Zhang
University of Southern California, USA

RIMS, Kyoto University, Japan

Emory University, USA

University of Oxford, UK

University of Notre Dame, USA

University of Minnesota, USA

Princeton University, USA

Brown University, USA

North Carolina State University, USA

Tata Inst. of Fund. Research, India

University of Michigan, USA

Stanford University, USA

Hasselt University, Belgium

Université Paris VII, France

Nihon University, Japan

University of California, San Diego, USA

Princeton University, USA

\section{PRODUCTION}

production@msp.org

Silvio Levy, Scientific Editor

See inside back cover or msp.org/ant for submission instructions.

The subscription price for 2015 is US $\$ 255 /$ year for the electronic version, and $\$ 440 /$ year ( $+\$ 55$, if shipping outside the US) for print and electronic. Subscriptions, requests for back issues and changes of subscribers address should be sent to MSP.

Algebra \& Number Theory (ISSN 1944-7833 electronic, 1937-0652 printed) at Mathematical Sciences Publishers, 798 Evans Hall \#3840, c/o University of California, Berkeley, CA 94720-3840 is published continuously online. Periodical rate postage paid at Berkeley, CA 94704, and additional mailing offices.

ANT peer review and production are managed by EditFLOW ${ }^{\circledR}$ from MSP.

\section{PUBLISHED BY}

- mathematical sciences publishers

nonprofit scientific publishing

http://msp.org/

(C) 2015 Mathematical Sciences Publishers 


\section{Algebra \& Number Theory}

Volume $9 \quad$ No. $4 \quad 2015$

Motivic Donaldson-Thomas invariants of small crepant resolutions

ANDREW MORRISON and KENTARO NAGAO

Étale homotopy equivalence of rational points on algebraic varieties

AMBRUS PÁL

Fermat's last theorem over some small real quadratic fields

NUNO FREITAS and SAMIR SIKSEK

Bounded negativity of self-intersection numbers of Shimura curves in Shimura surfaces MARTIN MÖLLER and DOMINGo TOLEDO

Singularities of locally acyclic cluster algebras

Angélica Benito, Greg Muller, Jenna Rajchgot and Karen E. Smith

On an analytic version of Lazard's isomorphism

GEORG TAMME

Towards local-global compatibility for Hilbert modular forms of low weight JAMES NEWTON

Horrocks correspondence on arithmetically Cohen-Macaulay varieties

981

Francesco Malaspina and A. Prabhakar RaO

The Elliott-Halberstam conjecture implies the Vinogradov least quadratic nonresidue conjecture

TERENCE TAO 